\title{
Transient ultrasonic guided wave simulation in layered composite structures using a hybrid wave and finite element scheme
}

\author{
Muhammad Khalid Malik ${ }^{\mathrm{a}, \mathrm{c}, *}$, Dimitrios Chronopoulos $^{\mathrm{a}}$, Gregor Tanner ${ }^{\mathrm{b}}$ \\ a Institute for Aerospace Technology 8 The Composites Group, University of Nottingham, NG7 2RD, United Kingdom \\ b School of Mathematical Sciences, University of Nottingham, NG7 2RD, United Kingdom \\ c Aernnova Engineering Division S.A., Madrid, 28034, Spain
}

\begin{abstract}
A guided wave simulation method for layered composites based on the wave and finite element scheme is presented. An approach for calculating complex displacement fields such as those generated from piezoelectric transducers is developed. The scattering of waves from different types of defects is computed. A rigorous energy based criteria is proposed for model order reduction. All calculations are carried out in the frequency domain and an inverse discrete fourier transform is performed to get the time domain result. Numerical examples of a multi-layered composite beam are performed to assess the performance and validate the methodology. Three types of damages are simulated namely a notch, a transverse crack and an internal delamination. The results are validated against finite element simulations and are found to be in excellent agreement. Moreover the approach is found to be orders of magnitude faster compared to finite element simulations.
\end{abstract}

Keywords: Guided Waves, Transient Analysis, Composites, SHM

\section{Introduction}

The pursuit of better performance and cost reduction has led to an increase in use of composite materials in aircraft structures. More than $50 \%$ of the structural components of the latest commercial jets from Airbus and Boeing are made of composites [1,2]. This is an achievement for the aerospace sector but it has resulted in a new set of challenges. Chief among them are the complex failure modes and mechanisms of composites [3. This presents a problem for effective integrity assessment of the structure through established structural health monitoring (SHM) techniques such as radiography [4], acoustic emission [5], ultrasonic waves [6] or eddy current [7]. Moreover, the potential presence of sub-surface damages such as delamination require techniques which can inspect the structures through the thickness.

\footnotetext{
* Corresponding Author

Email address: Muhammad.Malik@nottingham.ac.uk (Muhammad Khalid Malik)
} 
Ultrasonic guided waves have been demonstrated to be suitable for SHM in thin structures [8 10]. They can travel long distances, require minimal equipment and are sensitive to defects which makes them a costeffective SHM solution for composite structures [11 14]. The guided wave propagation characteristics of a structural waveguide contains information about the health of the structure, the presence of potential defects and their locations. In an SHM framework, this can be used to evaluate the structure by comparing the signal with a database of possible responses and by running a pattern recognition algorithm to detect the presence of damage [15-20]. Creating a database of patterns experimentally is often not practical, hence simulations are used to generate a library of baseline states. From here arises the need for fast and efficient simulation tools. The one-dimensional wave propagation has analytical solutions available for very simple waveguide geometries and structural discontinuities [21. But for composite structures with complex defects such as cracks and delaminations, the finite element (FE) method remains the state of the art in numerical simulations. FE is highly reliable for guided wave simulations but due to the very short wavelengths in the ultrasonic regime, a very high resolution in both spatial and temporal domains is required. Therefore the approach quickly becomes computationally challenging.

Over the years different methodologies have been developed to overcome this issue, the foremost being semi-analytical finite element method (SAFE) [22, 23. It uses FE to describe wave behaviour in the thickness direction whereas a complex exponential function is used to describe the displacement field in the direction of wave propagation [24]. In [25], a combined analytical FE approach (CAFA) for simulation of guided waves in isotropic structures has been developed based on the analytical models presented in [26]. CAFA uses a global analytical solution to model wave generation, propagation, scattering, mode conversion, and detection. It is highly efficient but unfortunately limited to isotropic structures. In order to tackle layered structures, the approach is extended by using the SAFE method. But the SAFE method cannot be used to model truly periodic structures and cannot be coupled with FE damage models [27]. Another recently developed method is scaled boundary finite element method (SBFEM) used for simulating guided waves and detecting damage in structures [28]. It has been shown to simulate guided waves efficiently and accurately in isotropic structures with defects. The dominant feature of these approaches is a combined analytical and numerical framework to reduce computational complexity. The wave and finite element (WFE) method is one such methodology developed in the last decade [29]. It is used in a number of research areas like structural identification [30, damage detection [31, multi-scale wave propagation [27] and wave steering in composites [32]. There have been efforts recently to develop a transient simulation method based on WFE [33, 34, but no method addressing ultrasonic guided waves exists so far.

The principal novelty introduced in this work is a WFE based approach for complete transient simulation of ultrasonic guided waves in composite structures. This contains a method for simulating complex displacement fields actuated by physical piezoelectric transducers. Also, a model order reduction strategy is presented for selecting the modes for wave propagation depending on the energy conservation across a 
finite sized defect in the structure which results in significant reduction in computation time. The proposed approach is capable of simulating guided wave propagation due to external excitation, scattering from complex damages, reflection from boundaries and time domain response at any point in the structure. The advantage of WFE over existing techniques is the capability of handling truly periodic structures as well as coupling with finite element models.

The paper is organised as follows. Sec. 2 contains the theoretical components of the methodology which includes the WFE scheme, modelling of coupling sections for arbitrary excitation, damage modelling and coupling to the main structure to simulate defects, a mode selection strategy to reduce the computational costs of the method and the complete transient domain signal reconstruction. Sec. 3 contains a series of examples to demonstrate the capabilities of the approach as well as the validation of results. This is followed by conclusions and future work recommendations in Sec. 4

\section{Nomenclature}

$\lambda^{+}, \lambda^{-} \quad$ Propagation constant

$\mathbf{a}^{+}, \mathbf{a}^{-} \quad$ Frequency domain amplitude vector

$\mathbf{D}, \mathbf{D}_{\mathbf{c}}, \mathbf{D}_{\mathbf{J}} \quad$ Dynamic stiffness matrix of waveguide, coupling section and damaged section

$\mathbf{f}_{\mathbf{c}}, \mathbf{f}_{\mathbf{c p}} \quad$ Internal and external forces for coupling section

$\mathbf{f}_{\mathbf{J}} \quad$ Internal forces for damaged section

$\mathbf{f}, \mathbf{f}_{\mathbf{p}} \quad$ Internal and external forces for waveguide

$\mathbf{K}_{\mathbf{c}}, \mathbf{M}_{\mathbf{c}}, \mathbf{C}_{\mathbf{c}}$ Stiffness, mass and damping matrices of coupling section

$\mathbf{K}_{\mathbf{J}}, \mathbf{M}_{\mathbf{J}}, \mathbf{C}_{\mathbf{J}}$ Stiffness, mass and damping matrices of damaged section

$\mathbf{K}, \mathbf{M}, \mathbf{C} \quad$ Stiffness, mass and damping matrices of waveguide

$\mathbf{q}, \mathbf{q}_{\mathbf{c}}, \mathbf{q}_{\mathbf{J}} \quad$ Nodal degrees of freedom of waveguide, coupling section and damaged section

R Rotation matrix

$\mathbf{S}, \tilde{\mathbf{S}} \quad$ Complete and reduced scattering matrices of damaged section

$\mathbf{T} \quad$ Transfer matrix of waveguide

$\omega \quad$ Angular frequency

$\phi_{f} \quad$ Wavemode eigenvector for $\mathbf{f}$

$\boldsymbol{\phi}_{q} \quad$ Wavemode eigenvector for $\mathbf{q}$

$c_{g} \quad$ Group velocity

$k \quad$ Wavenumber

$P, E \quad$ Power and energy

$\mathrm{S}_{0}, \mathrm{~A}_{0} \quad$ First symmetric and antisymmetric mode 


\section{Transient guided wave simulation}

The complete methodology for transient simulation of ultrasonic guided waves is presented in this section. The guided waves of interest in this work are the Lamb waves. Lamb waves are the constructive and destructive interferences and superposition of pressure wave and shear vertical wave undergoing multiple reflections between the traction free top and bottom surfaces of thin waveguides [35. A typical periodic structure with piezoelectric transducer (PZT) mounted on its surface is shown in Fig. 1. The PZT converts the electrical signal into mechanical waves where the wave mode type depends on the frequency of excitation. In the examples in Sec. 3 , the fundamental symmetric $\left(\mathrm{S}_{0}\right)$ and antisymmetric $\left(\mathrm{A}_{0}\right)$ Lamb modes are used. The approach presented here uses a divide and conquer strategy. The structure shown in Fig. 1 is divided into three sections: (i) a coupling section for arbitrary excitations, (ii) a damaged section containing arbitrary damage and (iii) the rest of the waveguide. The key to a fast and efficient method is to handle each of these sections separately and combining them together in a semi-analytical way using periodic structure theory and fundamental principles of continuity and equilibrium. The entire approach is illustrated schematically in Fig. 2. The WFE method is used to obtain wave propagation characteristics, wavenumbers and wavemodes, of the periodic waveguide. The externally excited amplitudes are obtained from the coupling section. The scattering matrix is obtained from the damaged section. Hence all the information necessary for calculating the transient response is complete. Also a rigorous energy conservation study is carried out to identify the modes relevant for reconstructing the response after scattering, in order to reduce the dimensionality of the overall problem.

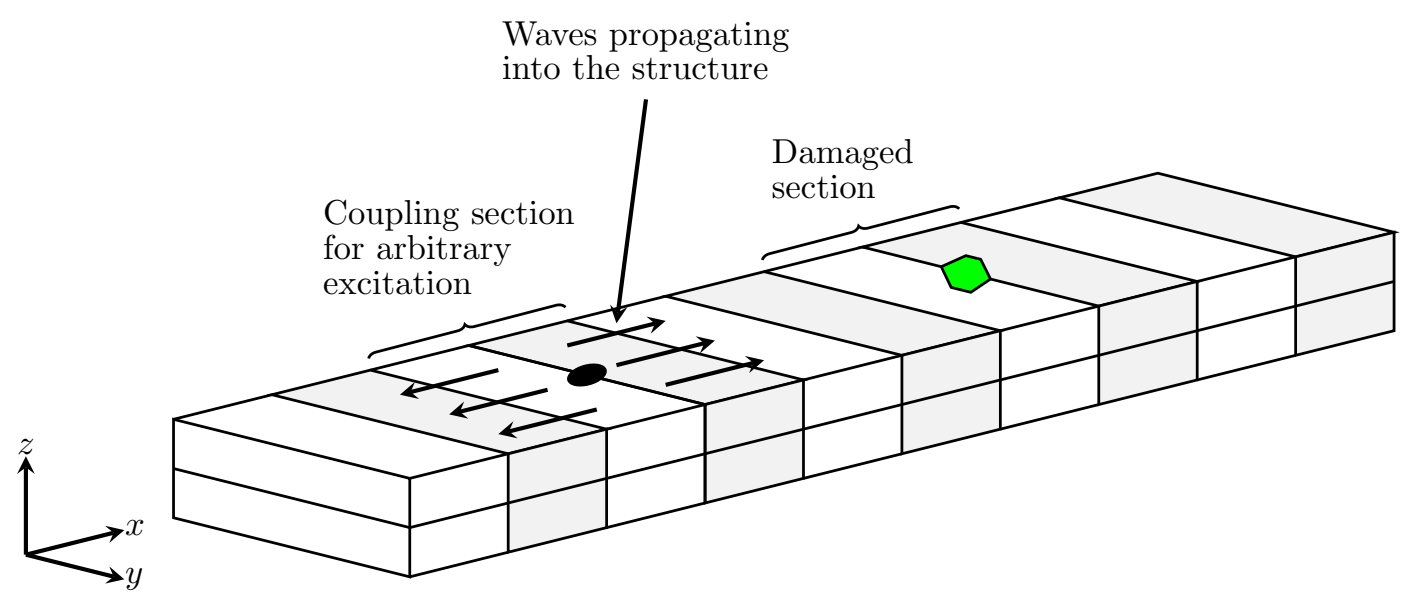

Figure 1: One dimensional periodic waveguide with external excitation and damage.

\subsection{Wave and finite element method}

The WFE method is used to obtain the wave propagation characteristics of the waveguide. This technique has been developed and applied in a number of domains over the last decade. Only a brief overview is 


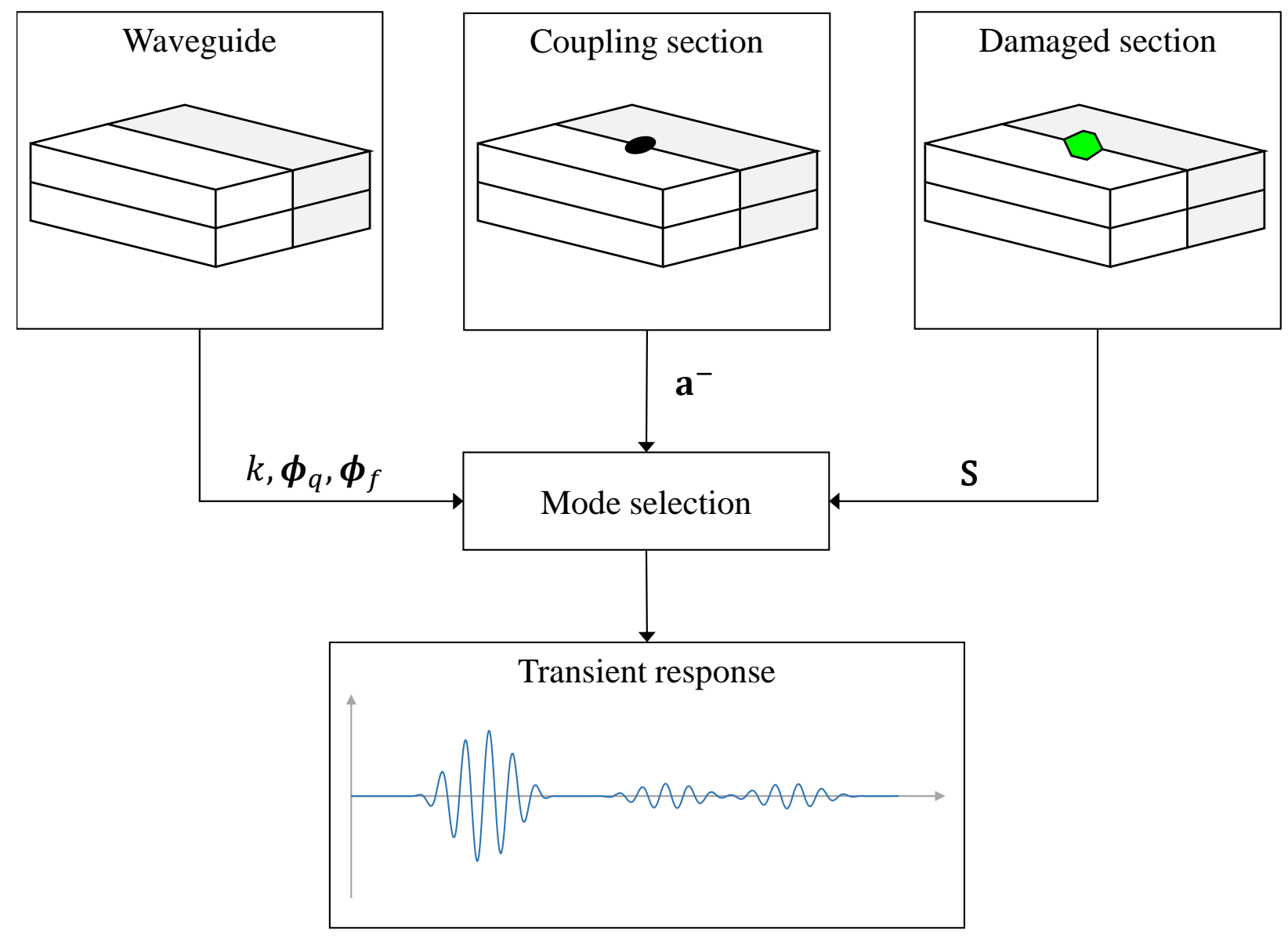

Figure 2: Schematic of the methodology for transient simulation of guided waves.

presented here, for more details refer to [36], 37]. The first step is to model a periodic section using an FE software. The cross-section can be of arbitrary complexity, hence making the method ideal for modelling layered media. It is necessary for the structure to be uniform in the direction of wave propagation and to have same number of degrees of freedom on the left $(L)$ and right $(R)$ hand side of the waveguide. The finite element model of the periodic section is used to obtain the stiffness $(\mathbf{K})$, mass (M) and damping (C) matrices to set up the dynamic equilibrium as follows:

$$
\begin{array}{r}
{\left[\mathbf{K}+i \omega \mathbf{C}-\omega^{2} \mathbf{M}\right] \mathbf{q}=\mathbf{f}+\mathbf{f}_{\mathbf{p}}} \\
\text { or } \quad \mathbf{D}(\omega) \mathbf{q}=\mathbf{f}+\mathbf{f}_{\mathbf{p}} .
\end{array}
$$

Here, $\mathbf{q}$ is the vector of nodal degrees of freedom internally partitioned into left and right interface degrees of freedom. $\mathbf{f}$ and $\mathbf{f}_{\mathbf{p}}$ are vectors of internal and external forces having the same dimension as $\mathbf{q}$. $\mathbf{D}(\omega)$ is the frequency dependent dynamic stiffness matrix which has blocks representing the interface and their interaction. In case there are internal degrees of freedom present in the waveguide, they can easily be 
condensed [38, 39]. In the absence of external forces, Eq. (1) has the following form:

$$
\left[\begin{array}{ll}
\mathbf{D}_{L L} & \mathbf{D}_{L R} \\
\mathbf{D}_{R L} & \mathbf{D}_{R R}
\end{array}\right]\left\{\begin{array}{c}
\mathbf{q}_{L} \\
\mathbf{q}_{R}
\end{array}\right\}=\left\{\begin{array}{c}
\mathbf{f}_{L} \\
\mathbf{f}_{R}
\end{array}\right\}
$$

where the subscripts $L$ and $R$ refer to the left and right sides of the interface. According to Bloch's theorem, the free wave propagation in a waveguide of length $l_{x}$ has the propagation constant $\lambda=\mathrm{e}^{-\mathrm{i} k l_{x}}$ which relates the nodal degrees of freedom on the left and right hand side by $\mathbf{q}_{R}=\lambda \mathbf{q}_{L}$ and $\mathbf{f}_{R}=-\lambda \mathbf{f}_{L}$. An eigenvalue problem for $\lambda$ is formulated by substituting this into Eq. (2), that is,

$$
\lambda\left\{\begin{array}{l}
\mathbf{q}_{L} \\
\mathbf{f}_{L}
\end{array}\right\}=\mathbf{T}\left\{\begin{array}{l}
\mathbf{q}_{L} \\
\mathbf{f}_{L}
\end{array}\right\},
$$

where

$$
\mathbf{T}=\left[\begin{array}{cc}
-\mathbf{D}_{L R}^{-1} \mathbf{D}_{L L} & \mathbf{D}_{L R}^{-1} \\
-\mathbf{D}_{R L}+\mathbf{D}_{R R} \mathbf{D}_{L R}^{-1} \mathbf{D}_{L L} & -\mathbf{D}_{R R} \mathbf{D}_{L R}^{-1}
\end{array}\right] .
$$

$\mathbf{T}$ is known as the transfer matrix. The propagation constants $\lambda$ are eigenvalues of $\mathbf{T}$. They exist in pairs of $\left[\lambda^{+}, \lambda^{-}\right]$for the positive and negative travelling waves, respectively. The eigenvalue problem can be poorly conditioned leading to numerical issues. Different formulations are used to mitigate this effect as described in [40]. The wavenumbers are calculated from the propagation constants. The eigenvectors $\phi$ are used as basis functions in the frequency domain. They exist for both positive $\boldsymbol{\phi}^{+}$and negative $\boldsymbol{\phi}^{-}$travelling waves. They are internally partitioned to represent displacement and forces as follows:

$$
\phi=\left\{\begin{array}{c}
\phi_{q} \\
\phi_{f}
\end{array}\right\}, \quad \text { where } \boldsymbol{\phi}^{+}=\left\{\begin{array}{c}
\boldsymbol{\phi}_{q}^{+} \\
\boldsymbol{\phi}_{f}^{+}
\end{array}\right\}, \boldsymbol{\phi}^{-}=\left\{\begin{array}{c}
\boldsymbol{\phi}_{q}^{-} \\
\boldsymbol{\phi}_{f}^{-}
\end{array}\right\} .
$$

\subsection{Inclusion of arbitrary excitation}

Piezoelectric transducers (PZT) are widely used to excite ultrasonic guided waves for SHM purposes. PZT converts electrical signals to physical displacements in the structure which can result in multi-modal wave propagation. Therefore the amplitudes generated in the waveguide are quite complex and need to be specified appropriately for reliable simulations. The technique presented here is capable of handling such complex displacement fields. Fig. 3illustrates the steps necessary to obtain the amplitudes generated in the structure due to external excitation. The physical signal in the time domain is transformed to the frequency domain by using discrete fourier transform (DFT) as follows:

$$
\mathbf{f}_{\mathbf{c p}}\left(\omega_{j}\right)=\sum_{i=1}^{m} \hat{\mathbf{f}}_{\mathbf{c p}}\left(t_{i}\right) \mathrm{e}^{-\mathrm{i} t_{i} \omega_{j}} \quad \text { where } j=1, \ldots, m,
$$

Here, $\hat{\mathbf{f}}_{\mathbf{c p}}$ is the time domain excitation signal sampled at $\mathrm{m}$ points and $\mathbf{f}_{\mathbf{c p}}$ is the frequency domain signal. The subscript $\mathbf{c}$ refers to the coupling section and $\mathbf{p}$ for the PZT excitation. The discretisation in frequency 
domain influences the resolution of the final transient response and hence should be chosen carefully. The coupling section is modelled and meshed using FE software. The stiffness $\left(\mathbf{K}_{\mathbf{c}}\right)$, mass $\left(\mathbf{M}_{\mathbf{c}}\right)$ and damping $\left(\mathbf{C}_{\mathbf{c}}\right)$ matrices are extracted from this model which are used to set up the dynamic equilibrium.

$$
\begin{array}{r}
{\left[\mathbf{K}_{\mathbf{c}}+\mathrm{i} \omega \mathbf{C}_{\mathbf{c}}-\omega^{2} \mathbf{M}_{\mathbf{c}}\right] \mathbf{q}_{\mathbf{c}}=\mathbf{f}_{\mathbf{c}}+\mathbf{f}_{\mathbf{c p}},} \\
\text { or } \mathbf{D}_{\mathbf{c}}(\omega) \mathbf{q}_{\mathbf{c}}=\mathbf{f}_{\mathbf{c}}+\mathbf{f}_{\mathbf{c p}} .
\end{array}
$$

$\mathbf{D}_{\mathbf{c}}(\omega)$ is the frequency dependent dynamic stiffness matrix and $\mathbf{q}_{\mathbf{c}}$ is the vector of nodal degrees of freedom for the coupling section while $\mathbf{f}_{\mathbf{c}}$ and $\mathbf{f}_{\mathbf{c p}}$ are internal and external forces acting on the coupling section. It is important to have the same degrees of freedom at the interface of the model and the waveguide. The degrees

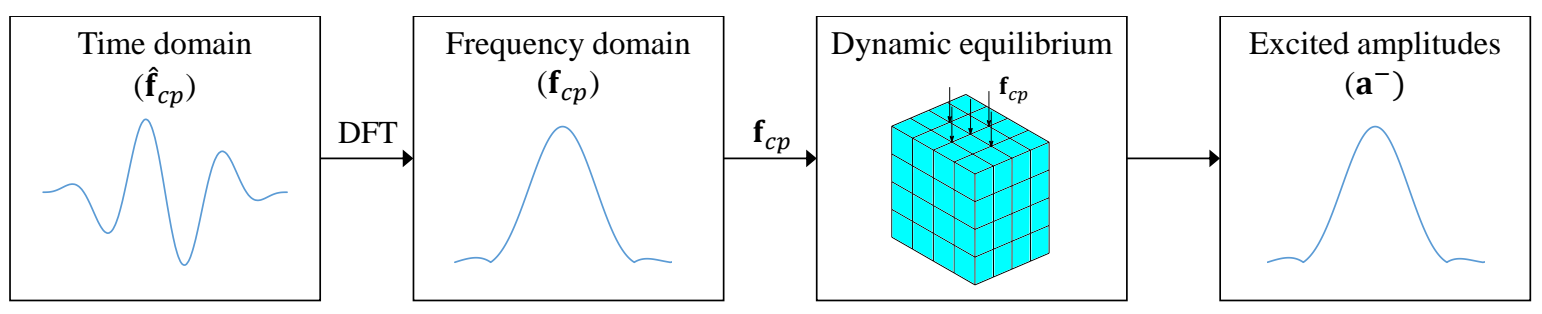

Figure 3: Schematic for obtaining amplitudes generated from external excitation.

of freedom of coupling section can be divided in 3 groups: (a) left and right hand interface degrees of freedom (ii), (b) internal degrees of freedom without external excitation (ni), and (c) internal degrees of freedom with external excitation ( $\mathbf{c p})$. Hence the degrees of freedom vector $\mathbf{q}_{\mathbf{c}}$ is internally partitioned between interface $\left(\mathbf{q}_{\mathbf{i i}}\right)$, non-interface-free $\left(\mathbf{q}_{\mathbf{n i}}\right)$ and non-interface-forced $\left(\mathbf{q}_{\mathbf{c p}}\right)$. The continuity and equilibrium conditions, shown in Eq. (8) must be satisfied at the interface between coupling section and the waveguide. This helps in introducing the wavemodes from Sec. 2.1] in dynamic equilibrium of the coupling section [41].

$$
\begin{aligned}
\mathbf{q}_{\mathbf{c}} & =\mathbf{R} \mathbf{q} \quad \text { (continuity), } \\
\mathbf{f}_{\mathbf{c}}-\mathbf{R f} & =\mathbf{0} \quad \text { (equilibrium). }
\end{aligned}
$$

Here, $\mathbf{R}$ is a rotation matrix to transform the degrees of freedom of the waveguide from their local coordinate system to a global coordinate system assuming that the coupling section degrees of freedom are already in a global coordinate system. The non-interface-free degrees of freedom must be condensed out of the equation of motion. The non-interface-forced degrees of freedom can either have prescribed forces $\left(\mathbf{f}_{\mathbf{c p}}\right.$ known) or prescribed displacements ( $\mathbf{q}_{\mathbf{c p}}$ known). In both cases, the prescribed quantities are factored out to the right hand side of the equation to act as a forcing function. This lends flexibility to the approach to cater for complex external excitations. Based on the approach presented in [41, a wave domain solution can be used to express the forces and displacements as follows:

$$
\begin{aligned}
& \mathbf{q}=\mathbf{R}\left[\boldsymbol{\phi}_{q}^{+} \mathbf{a}^{+}+\boldsymbol{\phi}_{q}^{-} \mathbf{a}^{-}\right], \\
& \mathbf{f}=\mathbf{R}\left[\boldsymbol{\phi}_{f}^{+} \mathbf{a}^{+}+\boldsymbol{\phi}_{f}^{-} \mathbf{a}^{-}\right] .
\end{aligned}
$$


Here the wavemodes become the basis function which express the frequency domain force and displacement as a linear combination of incoming $\left(\mathbf{a}^{+}\right)$and outgoing $\left(\mathbf{a}^{-}\right)$amplitudes. Guided wave based SHM set up has the structure completely at rest at the time of inspection. This prior knowledge is utilised to set the incoming amplitudes to zero. The end result is Eq. 10 which is solved for outgoing amplitudes in the structure as follows:

$$
\left(\tilde{\mathbf{D}}_{\mathbf{c}} \mathbf{R} \phi_{q}^{-}-\mathbf{R} \phi_{f}^{-}\right) \mathbf{a}^{-}=\mathbf{f}_{\mathbf{c p}}
$$

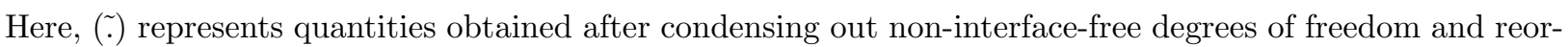
ganising the equation in the matrix vector form $\mathbf{A x}=\mathbf{b}$. This is another strength of this approach whereby Eq. 10 can be solved by any standard linear system of equations solver. This formulation also allows for exciting specific modes in the structure. The wavemode matrix $\phi_{q}$ obtained in Sec. 2.1 has the form $\left[\phi_{q 1} \phi_{q 2} \ldots . . \phi_{q n}\right]$ where each column is a vector representing a wavemode going to a maximum of $\mathrm{n}$ modes. Any single mode can be excited by obtaining its vector from the wavemode matrix, scaling it by the desired magnitude and then using it as prescribed displacement in the formulation. Similarly any combination of these modes can also be used by simply adding them together to simulate multi-modal wave propagation.

\subsection{Inclusion of arbitrary damage}

The discourse presented in this section closely follows [36] for the derivation of scattering matrix for joints of finite dimensions. When a propagating wave is incident on a discontinuity in a structure, it gives rise to reflected and transmitted waves. This is illustrated in Fig. 4 . The joint $(\mathbf{J})$ containing discontinuity is modelled in FE software from which the stiffness $\left(\mathbf{K}_{\mathbf{J}}\right)$, mass $\left(\mathbf{M}_{\mathbf{J}}\right)$ and damping $\left(\mathbf{C}_{\mathbf{J}}\right)$ matrices are obtained to establish the dynamic equilibrium as follows:

$$
\begin{array}{r}
{\left[\mathbf{K}_{\mathbf{J}}+\mathrm{i} \omega \mathbf{C}_{\mathbf{J}}-\omega^{2} \mathbf{M}_{\mathbf{J}}\right] \mathbf{q}_{\mathbf{J}}=\mathbf{f}_{\mathbf{J}}} \\
\text { or } \quad \mathbf{D}_{\mathbf{J}}(\omega) \mathbf{q}_{\mathbf{J}}=\mathbf{f}_{\mathbf{J}} .
\end{array}
$$

Here, $\mathbf{q}_{\mathbf{J}}$ and $\mathbf{f}_{\mathbf{J}}$ are vectors of nodal degrees of freedom and internal force vectors of the region containing the defect while $\mathbf{D}_{\mathbf{J}}(\omega)$ is the frequency dependent dynamic stiffness matrix. The interface degrees of freedom between the waveguides and the joint must be compatible to apply continuity and equilibrium conditions in the same way as shown in Eq. (8). It is important to state here that the waveguides surrounding the region of defect can be different from each other and may support different numbers of wavemodes. The formulation described here will still be able to obtain the amplitudes travelling into the structure as a result of scattering.

Once again the displacement and force vectors are expressed as a linear combination of wave amplitudes using Eq. (9). Substituting in Eq. (11) and condensing all non-interface degrees of freedom results in a relation for the scattering matrix $\mathbf{S}$ of a particular defect in the form of the ratios of incoming and outgoing 


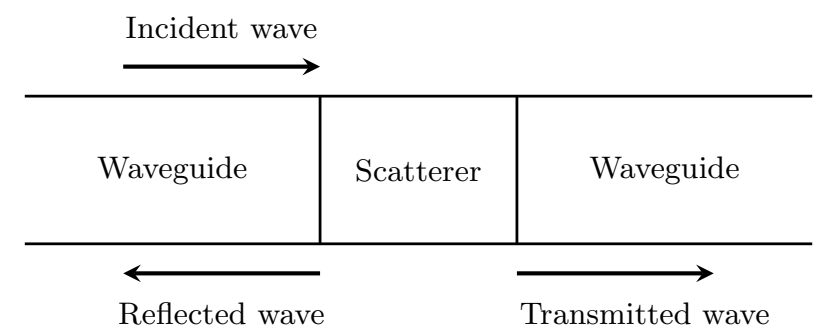

Figure 4: Scattering of waves from the defect.

amplitudes as follows:

$$
\mathbf{S}=-\left[\mathbf{R} \phi_{f}^{-}-\tilde{\mathbf{D}}_{J} \mathbf{R} \phi_{q}^{-}\right]^{-1}\left[\mathbf{R} \phi_{f}^{+}-\tilde{\mathbf{D}}_{J} \mathbf{R} \phi_{q}^{+}\right]
$$

Here, $\tilde{\mathbf{D}}_{J}$ is the condensed dynamic stiffness matrix of the joint. The scattering matrix for a damaged region connected on two sides by waveguides has the following structure:

$$
\mathbf{S}=\left[\begin{array}{ll}
\mathbf{S}_{11} & \mathbf{S}_{12} \\
\mathbf{S}_{21} & \mathbf{S}_{22}
\end{array}\right]
$$

Here, $\mathbf{S}_{11}$ is the matrix of reflection coefficients for waves incident from waveguide 1 and reflected back, $\mathbf{S}_{21}$ is the matrix of transmission coefficients for waves incident from waveguide 1 and transmitted to waveguide 2 and so on. Apart from reflection and transmission, these matrices contain mode conversion information as well. Hence, they form the basis of the mode selection strategy presented in Sec. 2.4 for time domain reconstruction. Only reflection is possible for waves incident on a boundary. Reflection from boundaries can also result in mode conversion of incident guided waves [42. Hence it is important to obtain the reflection matrix for each boundary present in the structure.

\subsection{Power and energy flow considerations}

The total energy flow and time averaged power associated with wave propagation in a structure provides important insight in the properties of wavemodes such as group velocity and the fundamental principle of energy conservation. The time averaged total energy of a wavemode $j$ is the sum of kinetic $\left(E_{k}\right)$ and potential $\left(E_{p}\right)$ energies [4]. The relations in terms of the wave basis are shown below:

$$
\begin{aligned}
& E_{k, j}=-\frac{1}{4} \omega^{2} \operatorname{Re}\left\{\boldsymbol{\phi}_{q, j}^{H} \mathbf{M} \boldsymbol{\phi}_{q, j}\right\}, \\
& E_{p, j}=\frac{1}{4} \operatorname{Re}\left\{\boldsymbol{\phi}_{q, j}^{H} \mathbf{K} \boldsymbol{\phi}_{q, j}\right\} .
\end{aligned}
$$

Here, (. $)^{H}$ denotes a Hermitian conjugate and $\boldsymbol{\phi}_{q, j}$ is the wavemode vector of nodal degrees of freedom for wavemode $j$. For an element length $\Delta$ in the propagation direction, the total time averaged energy per unit length is given as follows:

$$
\bar{E}_{j}=\frac{E_{k, j}+E_{p, j}}{\Delta} .
$$


The time averaged power $(P)$ associated with a propagating wave $j$ is obtained as follows:

$$
P_{j}=\frac{\omega}{2} \operatorname{Im}\left\{\boldsymbol{\phi}_{f, j}^{H} \boldsymbol{\phi}_{q, j}\right\}
$$

The group velocity $c_{g}$ for a guided wave is an important quantity. It is the speed at which energy propagates in a particular wave. It has a number of implications for setting up a guided wave simulation such as the maximum element size in wave propagation direction and the maximum time step increment allowed. The knowledge of $c_{g}$ as a function of frequency for a wavemode helps in fixing these parameters a priori with sufficient degree of confidence. It is obtained from the ratio of power and energy as follows:

$$
c_{g, j}=\frac{P_{j}}{\bar{E}_{j}} .
$$

Here, $c_{g, j}$ denotes the group velocity of wavemode $j$. It is important to note that $c_{g}$ only makes sense for a propagating wave as evanescent modes do not transfer any energy. In a multi-modal system where mode conversion also takes place, the power flow for a wave $j$ is equal to $\frac{1}{2} P_{j}\left|a_{j}\right|^{2}$ as given by [44]. Here $a_{j}$ is the amplitude of wave $j$. Considering a scattering matrix for a scatterer connected on both sides by a waveguide as shown in Fig. 5 , the scattering matrix can be segregated for the two waveguides as shown in Eq. (13). The energy conservation principle states that for an incident wave $j$ having reflected waves $i$ and transmitted wave $k$ the power scattering coefficients are calculated as follows:

$$
\mathcal{R}=\left[\mathcal{R}_{i j}\right]=\left[\left|r_{i j}\right|^{2} \frac{P_{i}}{P_{j}}\right], \quad \text { and } \mathcal{T}=\left[\mathcal{T}_{k j}\right]=\left[\left|t_{k j}\right|^{2} \frac{P_{k}}{P_{j}}\right] .
$$

The sum of power scattering coefficients is equal to unity in the absence of damping, that is,

$$
\sum_{i} \mathcal{R}_{i j}+\sum_{k} \mathcal{T}_{k j}=1 \quad \text { for each wavemode } j
$$

The power scattering coefficients for each wavemode indicate the energy they are carrying when a wave is scattered from a defect or boundary in a structure. This forms the basis of the model order reduction strategy. It is proposed that in the time domain reconstruction step only the modes carrying energy should be retained. This will reduce the problem size as it is shown in Sec. 3. where one or two main modes carry energy in the structure and will be sufficient for computing the complete transient response.

\subsection{Transient response reconstruction}

In order to obtain the complete time history at some arbitrary point in the structure, it is necessary to acquire all the amplitudes arriving at that point. Fig. 5illustrates a wave propagation example with the structure divided into waveguides, scatterer and coupling section. The goal is to obtain the response at the observation point for a desired time window. Each wave reaching the observation point has its own time of flight which is introduced as a phase change in frequency domain by multiplying the amplitude by $\mathrm{e}^{-\mathrm{i} k(\omega) x}$ where $x$ is the distance the wave has travelled and $k(\omega)$ is the frequency dependent wavenumbers for that 


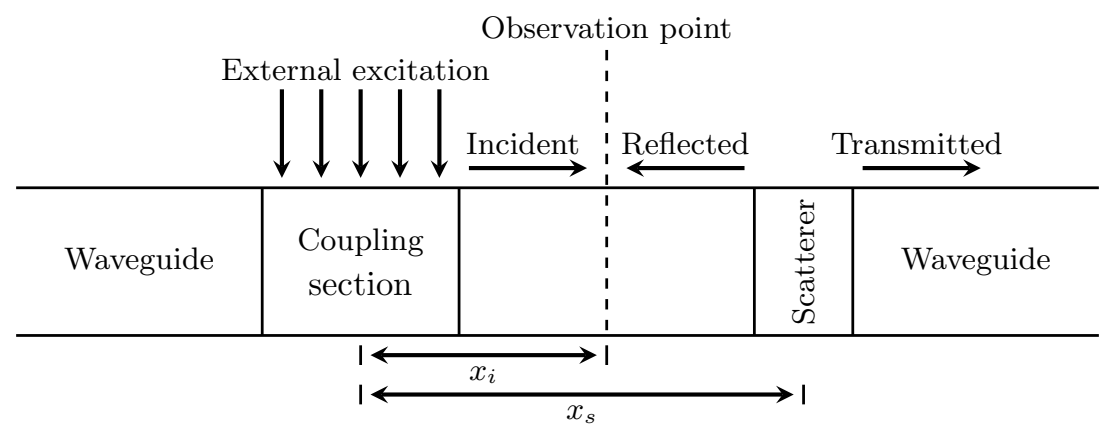

Figure 5: Wave propagation due to external excitation.

wave. The resulting amplitudes are still in frequency domain and they represent the wave motion of the entire cross-section. Assuming $n$ waves passing through the point of observation in a given time window, the $j^{\text {th }}$ amplitude is projected over the individual degrees of freedom by means of the basis functions as follows:

$$
\mathbf{q}_{j}=a_{j}^{+} \boldsymbol{\phi}_{q, j}^{+}+a_{j}^{-} \boldsymbol{\phi}_{q, j}^{-}
$$

Considering the illustration shown in Fig. 5 the first wave to reach the observation point will be the incident wave generated from the external excitation. After that, scattering will take place and the reflected amplitudes will also reach the observation point. These scattered amplitudes are calculated as follows:

$$
\tilde{\mathbf{a}}_{S}=\tilde{\mathbf{S}} \cdot \tilde{\mathbf{a}}_{I},
$$

Here, (.) represent the quantities obtained by performing the model order reduction presented in Sec. 2.4 The subscripts $I$ and $S$ refer to the incident and scattered amplitudes. The total response is obtained by summing up all the individual amplitudes reaching the observation point as shown below, that is,

$$
\mathbf{q}=\mathbf{q}_{1}+\mathbf{q}_{2}+\ldots .+\mathbf{q}_{n}
$$

where nodal displacements $\mathbf{q}$ of the waveguide are a function of frequency $\omega$. The final step is to perform an inverse DFT to obtain the time dependent displacement vector $\hat{\mathbf{q}}$, that is,

$$
\hat{\mathbf{q}}\left(t_{j}\right)=\frac{1}{m} \sum_{i=1}^{m} \mathbf{q}\left(\omega_{i}\right) \mathrm{e}^{-\mathrm{i} \omega_{i} t_{j}} \quad \text { where } j=1, \ldots, m,
$$

where $m$ is the sampling frequency used in Sec. 2.2 to go from the time domain to the frequency domain.

\section{Numerical Examples}

In this section, numerical examples are presented of a multi-layered composite beam with different types of damages. The results are compared with full FE simulations performed in Abaqus and WFE simulations 
done in MATLAB. The method presented in Sec. 2 is capable of handling the material damping but it is ignored in the following examples for simplicity. The layup under consideration is a 6 layered carbon fibre beam with $\left[0_{2} / 90_{2} / 0_{2}\right]$ stacking sequence. A single carbon fibre layer has a density of $1560 \mathrm{~kg} / \mathrm{m}^{3}$ and the stiffness matrix as shown in Eq. 24] which is obtained from [25], that is,

$$
C=\left[\begin{array}{cccccc}
143.8 & 6.2 & 6.2 & 0 & 0 & 0 \\
6.2 & 13.3 & 6.5 & 0 & 0 & 0 \\
6.2 & 6.5 & 13.3 & 0 & 0 & 0 \\
0 & 0 & 0 & 3.6 & 0 & 0 \\
0 & 0 & 0 & 0 & 5.7 & 0 \\
0 & 0 & 0 & 0 & 0 & 5.7
\end{array}\right] G P a
$$

The forced excitation in all the subsequent examples will be a Hanning windowed sinusoid. This excitation function ensures minimum dispersion around a central frequency due to the narrow bandwidth of the signal. The time domain forcing function for FE simulations is shown in Fig. 6a and its frequency domain transform is shown in Fig. $6 \mathrm{~b}$. The signal has a central excitation frequency of $100 \mathrm{kHz}$. The amplitude for the $\mathrm{S}_{0}$ mode is kept higher than the $\mathrm{A}_{0}$ mode. This results in observable out-of-plane amplitudes due to the Poisson effect from $\mathrm{S}_{0}$ excitation. Naturally, it would be better to keep more cycles to ensure minimum spread in the frequency domain. However, that increases the length of the signal in the time domain which needs to be kept to a reasonable time limit to ensure that the incident and reflected signals are separately identifiable in post processing.

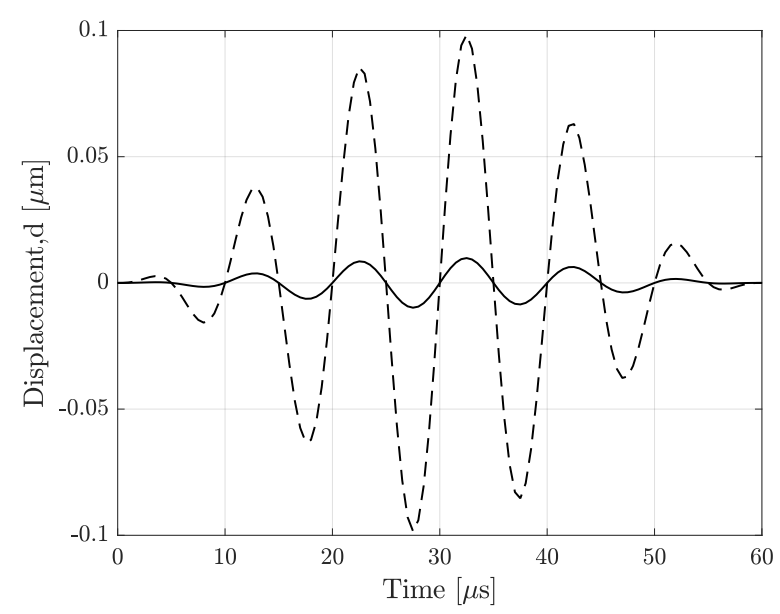

(a) Time domain

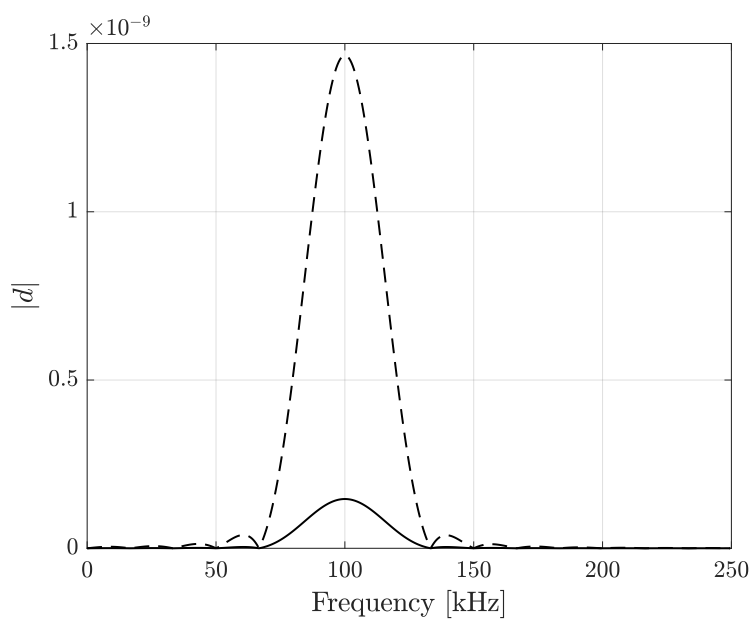

(b) Frequency domain

Figure 6: Excitation signal used for $\mathrm{S}_{0}(--)$ and $\mathrm{A}_{0}$ modes (-) respectively.

A multi-layered carbon fibre composite beam with length $l$, width $w$ and height $h$ is shown in Fig. 7 
The excitation is applied at one end of the beam and the response is observed at two different locations, one before and one after the damage. In each case, a thorough study of the wave propagation velocities and respective wavelengths will be carried out to ensure that the results are mesh independent and the FE simulation is not performed with an overly fine mesh. This would result in a fair comparison between FE and WFE results. All simulations in this section are performed on the same computer with a $7^{\text {th }}$ Generation Inter Core i3-7100U 2.50 GHz processor and 8 GB RAM.

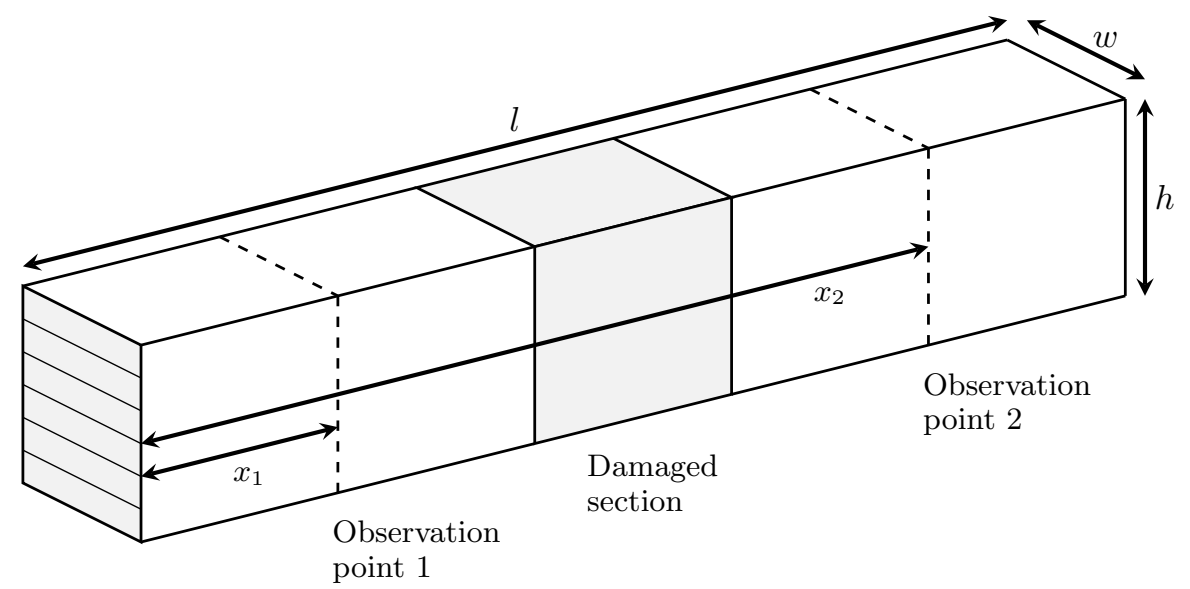

Figure 7: Carbon fibre composite beam with damage.

\subsection{Waveguide modelling}

Important considerations for modelling the waveguide are presented in this section. The waveguide is the representative section of the structure from which the wavenumbers and wavemodes are extracted as presented in Sec. 2.1. The group velocity of the desired modes directly influence the element length of the waveguide mesh and minimum time step size for the FE, that is,

$$
\lambda=\frac{2 \pi}{k(\omega)}, \quad \Delta \leq \frac{\lambda}{10}, \quad \Delta t<\frac{\Delta}{c_{g}(\omega)},
$$

where $\lambda$ is the wavelength, $\Delta$ is the element length in propagation direction and $\Delta t$ is the time step for the explicit FE simulation. For all subsequent examples the waveguide is modelled with C3D8 brick elements from the standard Abaqus element library [45. This is a general purpose solid continuum element with 8 nodes and 3 degrees of freedom at each node. The cross-section is discretrised with 6 elements through the thickness and 4 elements across the width. It is necessary to have at least 6 elements in the thickness direction as these elements are prone to shear locking which leads to incorrect results for the out-of-plane $\mathrm{A}_{0}$ mode. For a central excitation frequency of $100 \mathrm{kHz}, \Delta$ is chosen to be $0.2 \mathrm{~mm}$. The material properties are taken from Eq. (24). This discretisation results in 210 degrees of freedom for the waveguide with 105 positive and 105 negative modes. Most of these modes are evanescent in nature. They are important for 
energy conservation in the event of scattering from a defect. The two main propagating modes which will be used in the following sections are shown in Fig. 8a. They are the fundamental symmetric $\left(\mathrm{S}_{0}\right)$ and antisymmetric $\left(\mathrm{A}_{0}\right)$ Lamb modes.

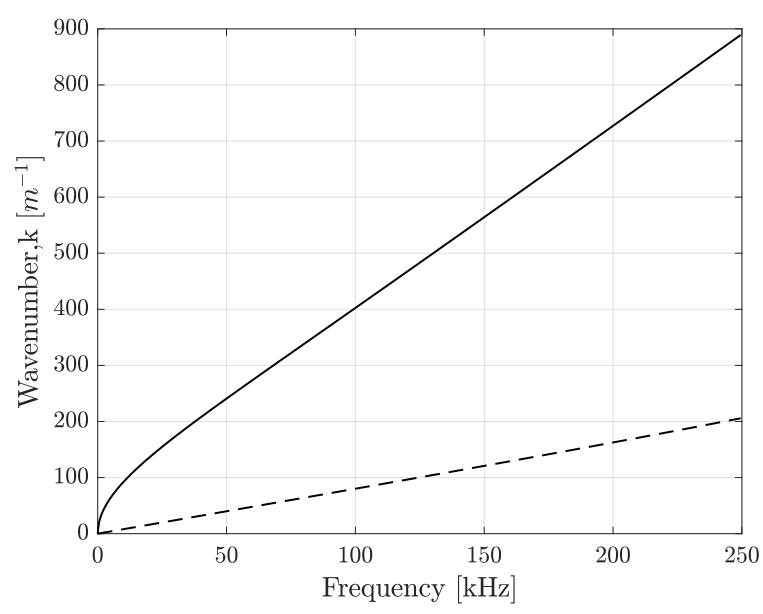

(a) Dispersion curves

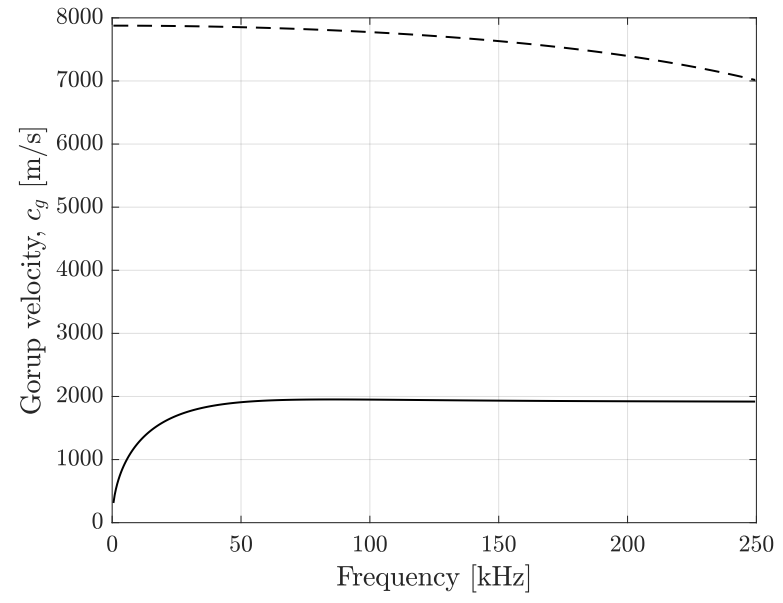

(b) Group velocity

Figure 8: Dispersion curves and group velocity for $\mathrm{S}_{0}(--)$ and $\mathrm{A}_{0}$ modes (-) for a 6 layer $\left[0_{2} / 90_{2} / 0_{2}\right]$ carbon fibre beam.

\subsection{Multi-modal wave propagation}

In this section a trivial case of Fig. 7 will be simulated where the beam is undamaged. The goal is to demonstrate the ability of WFE method to simulate multi-modal wave propagation in composite structures. The beam has the following dimensions: $l=0.55 \mathrm{~m}, w=2 \mathrm{~mm}, h=4 \mathrm{~mm}$. Only one observation point is selected at $x_{1}=0.25 \mathrm{~m}$, such that the different modes can be identified. A combined boundary condition of in-plane and out-of-plane displacement is applied on the left face in Fig. 7 to excite the $S_{0}$ and $A_{0}$ modes simultaneously. In the absence of damage, scattering does not take place and waves simply propagate through the beam. The result is shown in Fig. 9 where two different wavemodes are visible. The results from FE and WFE method are found to be well in agreement with each other.

\subsection{Composite beam with damage}

In this section three different types of damage will be considered, namely a notch, a crack and a delamination as illustrated in Fig. 10. The material properties and the layup will be the same as in Section 3.2 for all of the cases. Also the same excitation functions will be used. The problem set up is as shown in Fig. 7 where the damaged section is modified for each case. First a scattering matrix is calculated and an energy conservation study is carried out. The modes resulting in the largest reflected amplitude are used for identifying that damage and only the modes carrying energy are retained for response reconstruction. This 


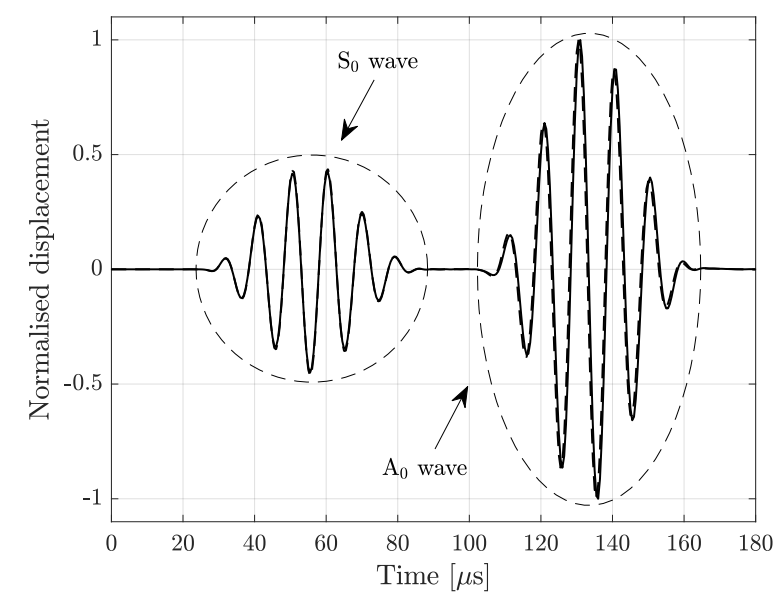

Figure 9: Out-of-plane displacement of a corner node at the observation point. The time domain response comparison between FE (-) and WFE (- -).

model order reduction strategy leads to a significant decrease in memory consumption and computation time in comparison to FE simulations. It also demonstrates the capability of the WFE approach to simulate complex damage scenarios in one-dimensional composite structures.

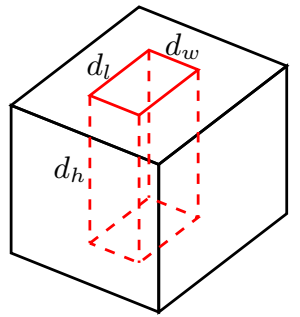

(a) Notch.

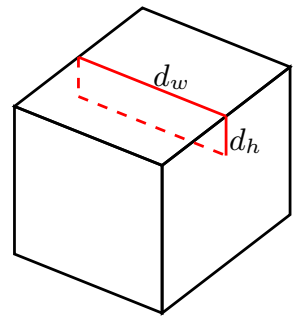

(b) Crack.

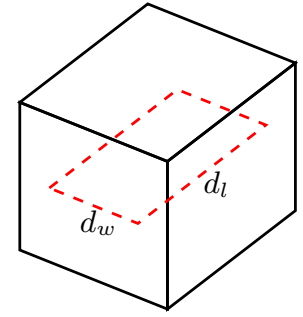

(c) Delamination.

Figure 10: Types of damages simulated.

\subsubsection{Notch damage}

Firstly, an example with a rectangular notch in the centre of the damaged section is considered. The beam has the following dimensions: $l=1 \mathrm{~m}, w=2 \mathrm{~mm}, h=4 \mathrm{~mm}$ with a damaged section at $x_{s}=0.5 \mathrm{~m}$ and the two observation points at $x_{1}=0.2 \mathrm{~m}$ and $x_{2}=0.7 \mathrm{~m}$. The dimensions of the notch are $d_{w}=1.2 \mathrm{~mm}$, $d_{l}=1 \mathrm{~mm}$ and $d_{h}=4 \mathrm{~mm}$. Such a discontinuity in the structure will lead to scattering of any propagating wave. Since it is possible to detect this hole by means of either the $\mathrm{S}_{0}$ or $\mathrm{A}_{0}$ wave, a study of the scattering coefficients is carried out to select the best mode for identification.

The reflection and transmission coefficients for the $S_{0}$ and $A_{0}$ modes around the central frequency of 


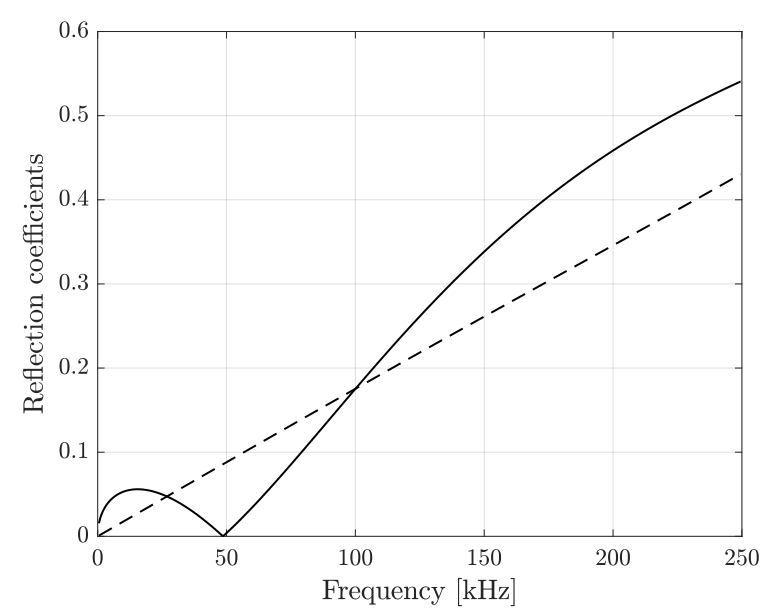

(a) Reflection coefficient

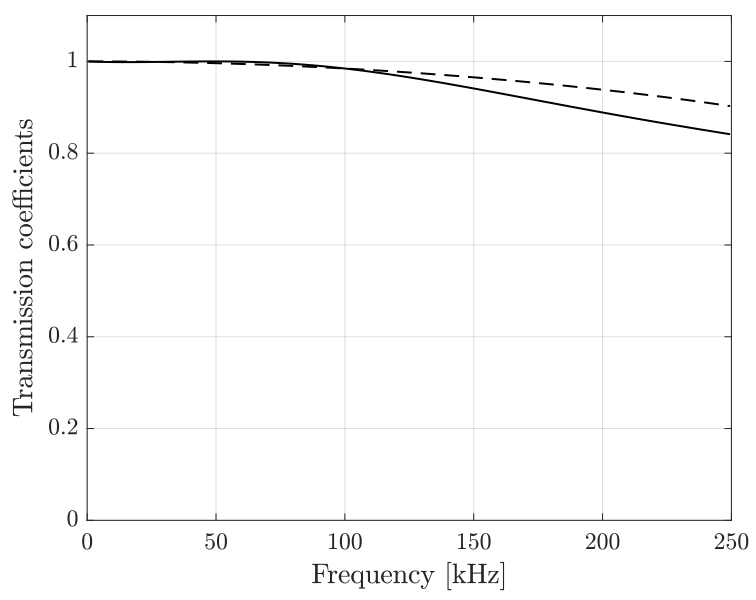

(b) Transmission coefficient

Figure 11: Scattering coefficients for a rectangular hole for $\mathrm{S}_{0}$ to $\mathrm{S}_{0}(--)$ and $\mathrm{A}_{0}$ to $\mathrm{A}_{0}(-)$.

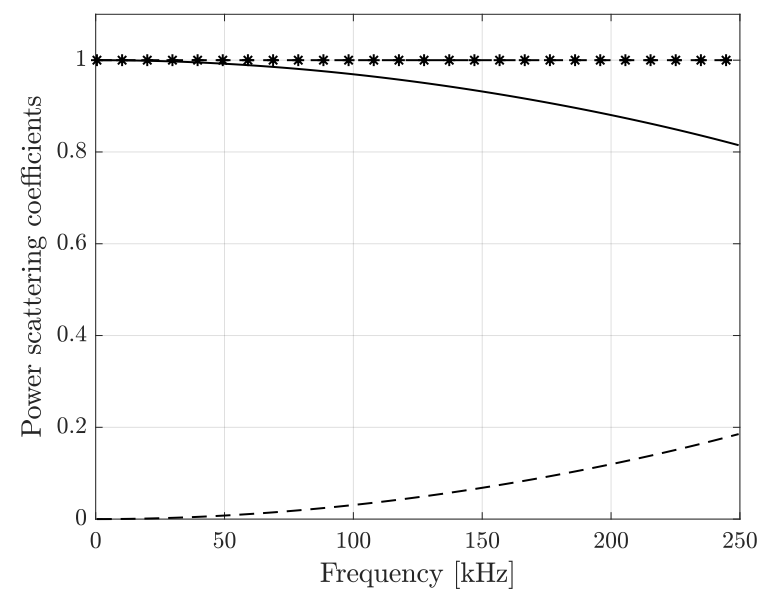

(a) Incident $\mathrm{S}_{0}$ wave

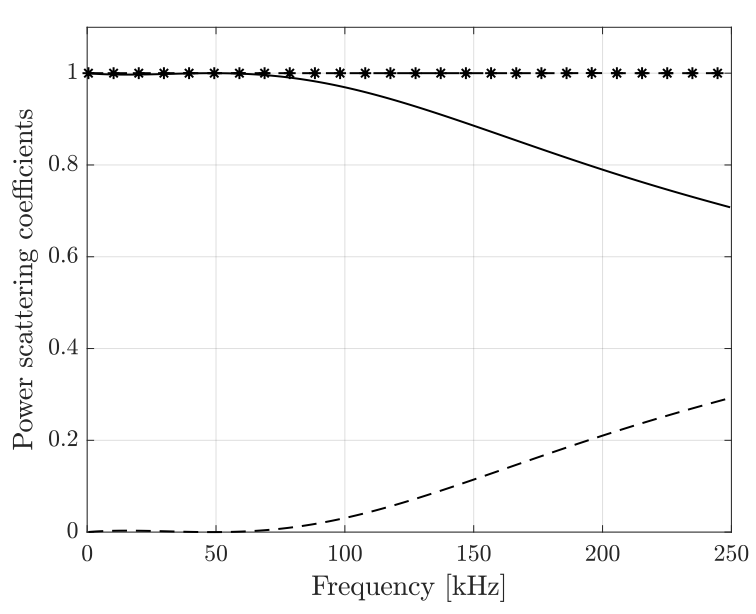

(b) Incident $A_{0}$ wave

Figure 12: Energy conservation for a beam with rectangular hole for incident $\mathrm{S}_{0}$ and $\mathrm{A}_{0}$ modes with reflection coefficients ($-)$, transmission coefficients $(-)$ and the total sum $\left(-^{*}\right)$.

excitation of $100 \mathrm{kHz}$ are almost the same as can be seen in Figs. 11a and 11b. This makes them both suitable candidates for identifying this damage. The power scattering coefficients for both modes are plotted in Figs. $12 \mathrm{a}$ and $12 \mathrm{~b}$. All other modes are suppressed here as only the $\mathrm{S}_{0}$ and $\mathrm{A}_{0}$ modes carry energy. This also means that mode conversion does not occur due to this discontinuity in the structure. This permits the use of only the respective reflection and transmission coefficients for reconstructing response at the point of observation. Since neither of the modes seem to have an advantage over the other, the $\mathrm{S}_{0}$ mode is arbitrarily 
selected. This leads to a spatial discretisation less finer than the one required for $\mathrm{A}_{0}$ mode as it has a lower wavenumber around $100 \mathrm{kHz}$, see Fig. 8a. The $\mathrm{S}_{0}$ mode has a significantly high velocity, see Fig. $8 \mathrm{~b}$ and hence requires a fine temporal discretisation with a stable time step $\Delta t$ of $1 \times 10^{-8} \mathrm{~s}$. The simulation in Abaqus is done using C3D8R reduced integration elements with a total of 80000 elements. A time window of $230 \mu \mathrm{s}$ is simulated. The FE simulation takes 2239s. The simulation using WFE approach is carried out using the same periodic section as in Sec. 3.2 Scattering matrix of the hole is calculated and stored in memory which can be reused for different spatial configurations. The total time taken for generating response at the two observation points is 0.1922 s. The comparison of the results from FE and WFE approach is shown in Figs. 13 and 14 .

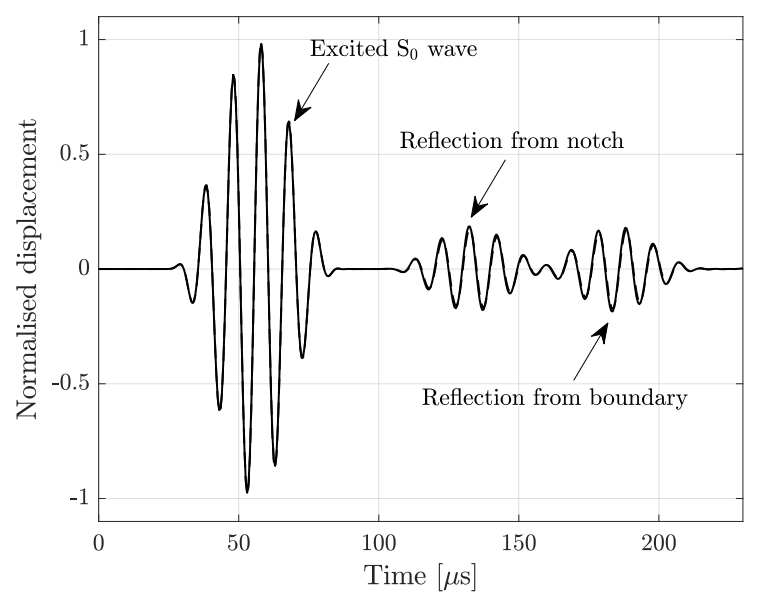

(a) In-plane

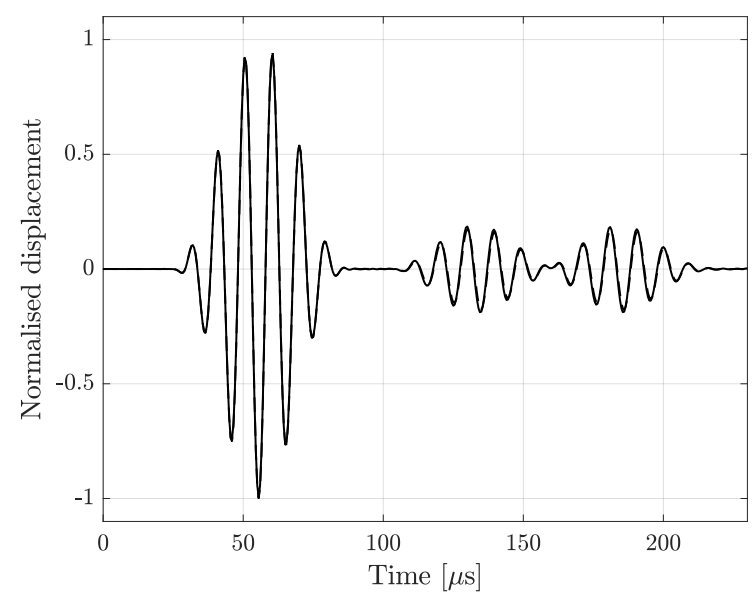

(b) Out-of-plane

Figure 13: Time domain comparison between FE (-) and WFE (- -) at a node located at observation point 1 for a beam with a notch damage.

The plots show the in-plane and out-of-plane displacements at the points of observation normalised to the absolute maximum of the displacement at the excitation point. The reflected amplitudes from the notch are observable in Fig. 13a. It is crucial to capture these reflected amplitudes accurately to develop an identification strategy. The transmission coefficients around the excitation frequency are slightly below 1 as shown in Fig. 11b, Therefore the wave amplitude is reduced on crossing the notch as shown in Fig. 14a,

\subsubsection{Crack}

Following the strategy presented in the previous section, the transient response of a composite beam with a crack damage will be simulated in this section. The beam has the same dimensions as in Sec. 3.3.1 while the crack is located at $x_{s}=0.4 \mathrm{~m}$. The observation points are located at $x_{1}=0.16 \mathrm{~m}$ and $x_{2}=0.55 \mathrm{~m}$. The crack is modelled by node duplication. The dimensions of the crack are $d_{w}=0.2 \mathrm{~mm}$ and $d_{h}=0.05$ 


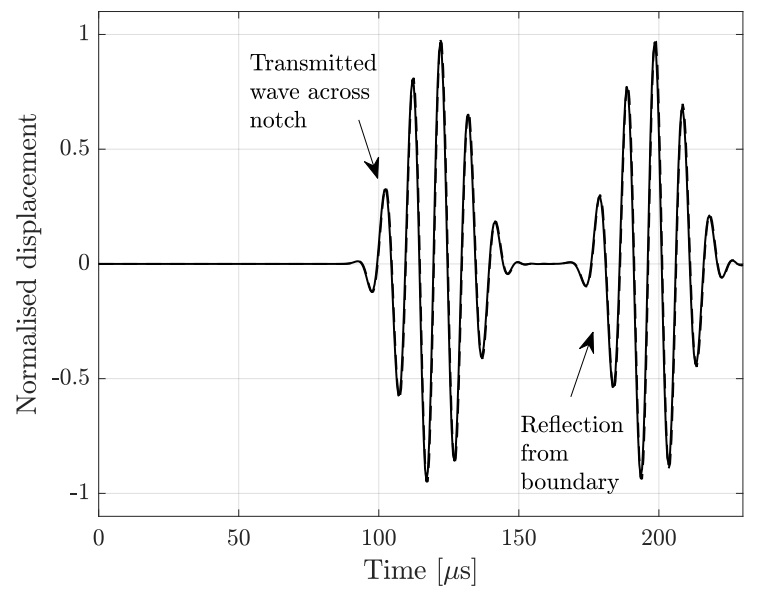

(a) In-plane

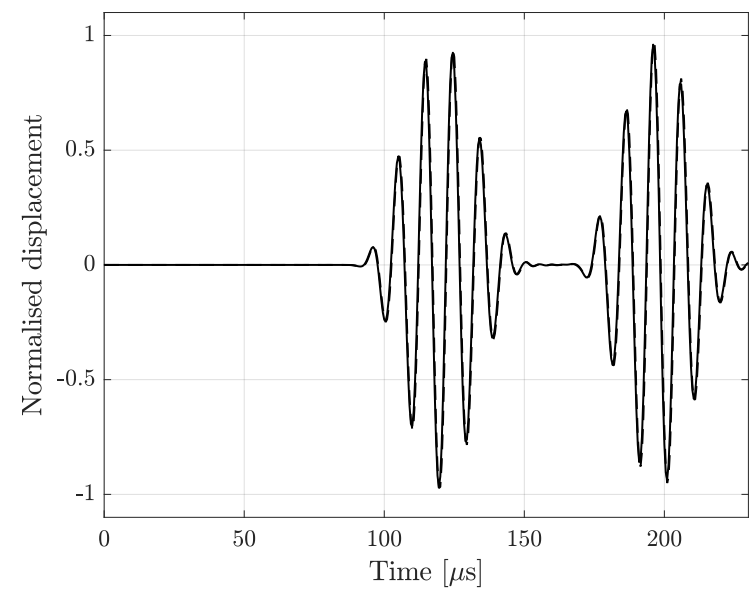

(b) Out-of-plane

Figure 14: Time domain comparison between FE (-) and WFE (- -) at a node located at observation point 2 for a beam with a notch damage.

mm. The crack is located in the top most zero degree layer as shown in Fig. 10.

The comparison of the scattering coefficients is performed to select the appropriate mode for detecting the crack. The coefficients are plotted in Figs. $15 \mathrm{a}$ and $15 \mathrm{~b}$. The reflection coefficients for $\mathrm{A}_{0}$ to $\mathrm{A}_{0}$ are slightly higher than $\mathrm{S}_{0}$ to $\mathrm{S}_{0}$ but are still of the same order of magnitude. The transmission coefficients also show similar values for both the modes. Looking at Figs. 16a and 16b it is observed that modes other than $\mathrm{S}_{0}$ to $\mathrm{S}_{0}$ and $\mathrm{A}_{0}$ to $\mathrm{A}_{0}$ are also carrying energy. In case of an incident $\mathrm{S}_{0}$ mode, these are the mode converted reflection and transmission to $\mathrm{A}_{0}$ mode and vice versa for the incident $\mathrm{A}_{0}$ mode. Their coefficients have significant amplitudes as can be seen in Figs. $15 \mathrm{a}$ and $15 \mathrm{~b}$. Since both modes result in similar amplitudes, the $\mathrm{S}_{0}$ mode is arbitrarily selected. This leads to a considerably shorter time window for the simulation as $\mathrm{S}_{0}$ wave propagates faster than the $\mathrm{A}_{0}$ wave. Note that the power scattering coefficients sum up to one. For convenience, only the reflection coefficients are shown in Figs. 16a and 16b. These are two orders of magnitudes smaller than the transmission coefficients and would hardly be visible plotting all coefficients simultaneously.

The mesh in the thickness direction is refined to capture the mode converted $\mathrm{A}_{0}$ wave accurately. The FE simulation is carried out using 120000 C3D8R elements. The time taken for the simulation is 3139 seconds. Model complexity in WFE approach is reduced by retaining only the $\mathrm{S}_{0}$ to $\mathrm{S}_{0}$ reflection and transmission and $\mathrm{S}_{0}$ to $\mathrm{A}_{0}$ reflection and transmission. Once the modes, basis functions and scattering coefficients are loaded into the memory, the reconstruction takes 0.3654 seconds. Similarly as before, it is possible to simulate multiple damage configurations without incurring any time penalty for loading the data. The results from FE and WFE are shown in Figs. 17 and 18 for the in-plane and out-of-plane displacements. Fig. 17a shows 


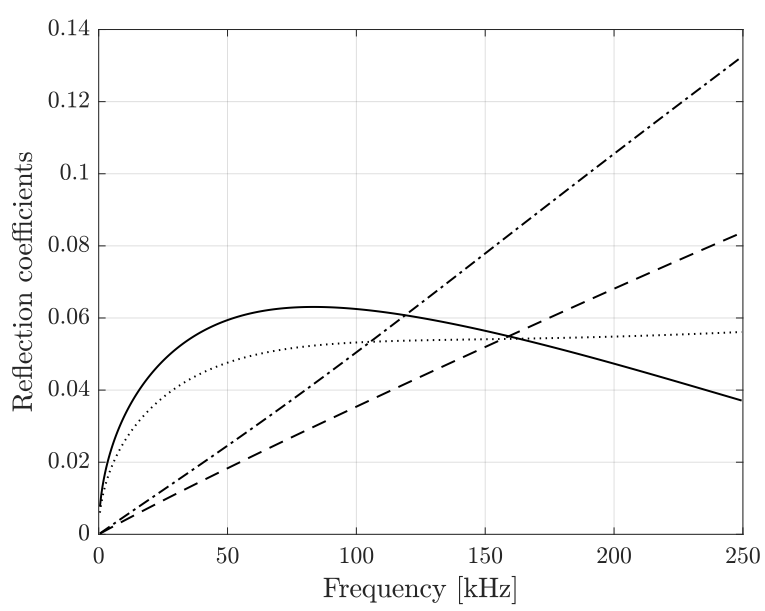

(a) Reflection coefficient

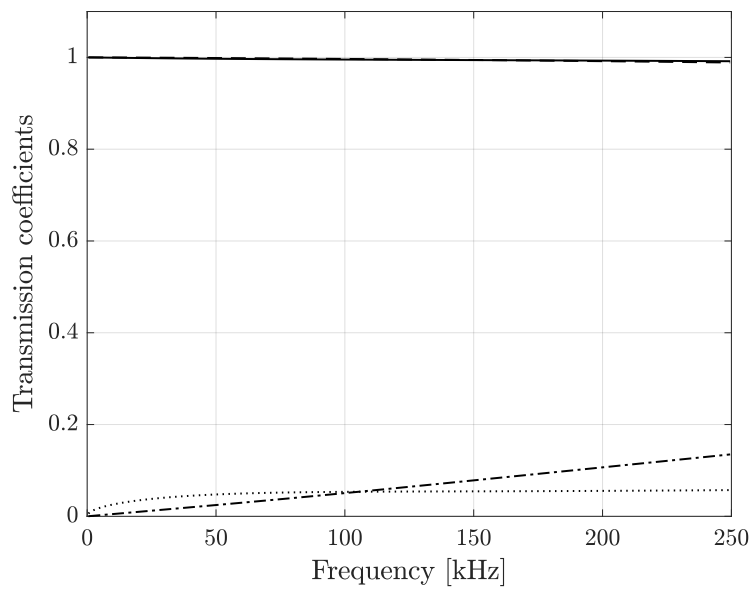

(b) Transmission coefficient

Figure 15: Scattering coefficients for a crack in a beam. $S_{0}$ to $S_{0}(--), S_{0}$ to $A_{0}(\cdots), A_{0}$ to $A_{0}(-)$ and $A_{0}$ to $S_{0}(-\cdot-)$.

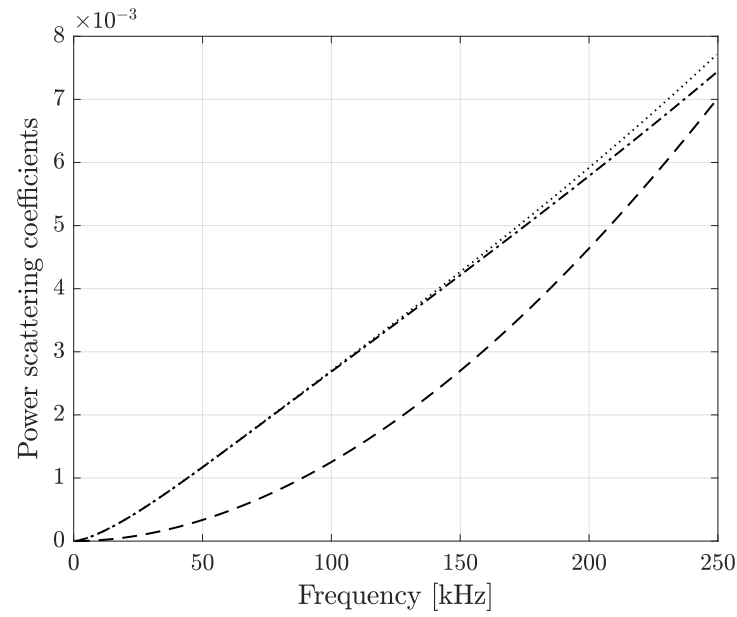

(a) Incident $\mathrm{S}_{0}$ wave

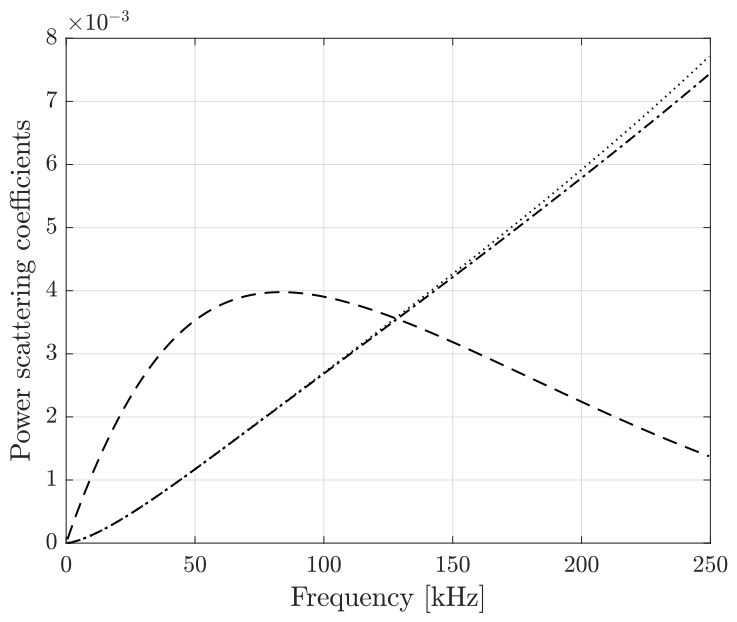

(b) Incident $A_{0}$ wave

Figure 16: Energy carrying modes for a beam with a crack with $S_{0}$ to $S_{0}$ and $A_{0}$ to $A_{0}$ reflection coefficients (- -), $S_{0}$ to $A_{0}$ and $A_{0}$ to $S_{0}$ reflection (---) and transmission coefficients ( ...). $S_{0}$ to $S_{0}$ and $A_{0}$ to $A_{0}$ transmission coefficients are not plotted as they are two orders of magnitude larger.

three distinctly identifiable reflections at observation point 1 , two for the $\mathrm{S}_{0}$ mode and one for the $\mathrm{A}_{0}$ mode. The out-of-plane displacement in Fig. $17 \mathrm{~b}$ show the distinction between the two modes clearly with the $\mathrm{A}_{0}$ mode having significantly larger amplitude. The same case occurs for observation point 2 where the transmitted wave has both the $S_{0}$ and the $A_{0}$ wave. The results are found to be in good agreement. 


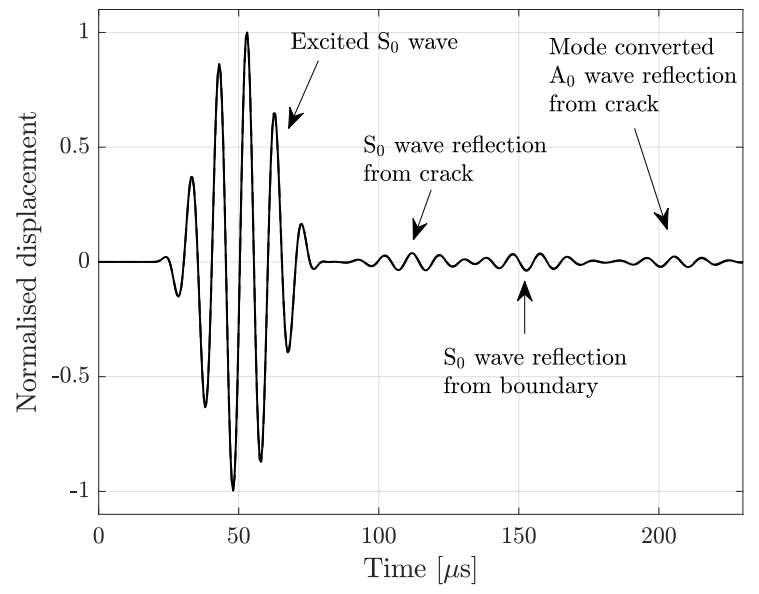

(a) In-plane

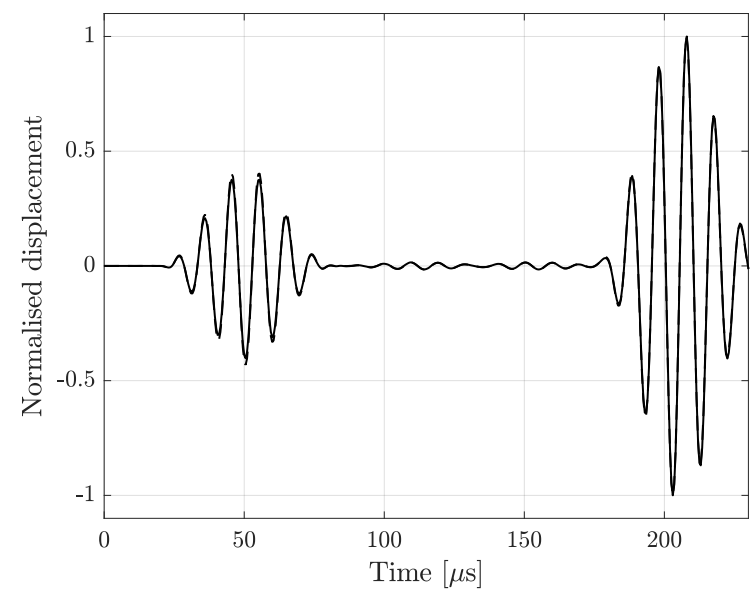

(b) Out-of-plane

Figure 17: Time domain comparison between FE (-) and WFE (- -) at a node located at observation point 1 for a beam with a crack damage.

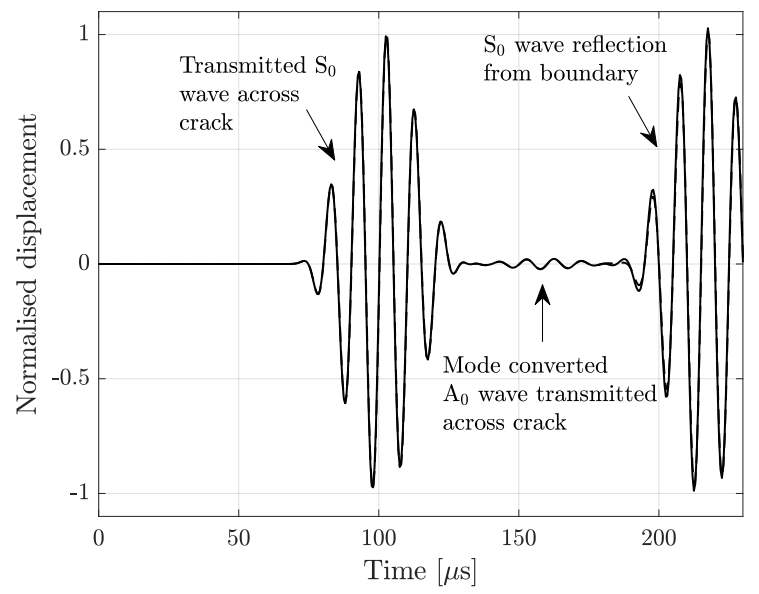

(a) In-plane

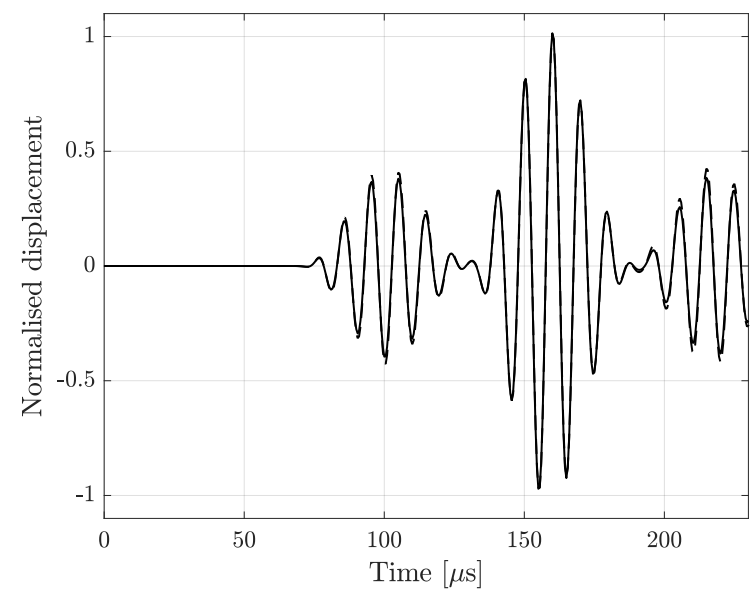

(b) Out-of-plane

Figure 18: Time domain comparison between FE (-) and WFE (- -) at a node located at observation point 2 for a beam with a crack damage.

\subsubsection{Delamination}

In this section a delamination is simulated in a composite beam. The delamination is introduced between the second and the third layer from the top. The dimensions of the delamination are $d_{l}=2 \mathrm{~mm}$ and $d_{w}=2 \mathrm{~mm}$. It is modelled by node duplication between two layers. The length of the beam is $l=0.6 \mathrm{~m}$ while the centre of delamination is at $x_{s}=0.2998 \mathrm{~m}$. The observation points 1 and 2 are located at 
$x_{1}=0.15 \mathrm{~m}$ and $x_{2}=0.45 \mathrm{~m}$.

Once again the mode selection study is performed by obtaining the scattering coefficients for the delamination. The results are shown in Figs. 19a and 19b. An order of magnitude difference between the reflection coefficients for $S_{0}$ to $S_{0}$ and $A_{0}$ to $A_{0}$ is observed. This is consistent with what is mentioned in the literature, that is, delamination is easier to detect with $\mathrm{A}_{0}$ waves [46]. There is also mode conversion taking place giving significant amplitudes for $S_{0}$ to $A_{0}$ and $A_{0}$ to $S_{0}$ modes. Both these facts are also supported by Figs. 20a and $20 \mathrm{~b}$ which show the main modes carrying energy. Hence, the $\mathrm{A}_{0}$ mode is selected to detect delamination. $\mathrm{A}_{0}$ to $\mathrm{A}_{0}$ reflection and transmission coefficients and $\mathrm{A}_{0}$ to $\mathrm{S}_{0}$ reflection and transmission coefficients are retained in the reduced model. Note that the power scattering coefficients sum up to one; for convenience, only the reflection coefficients are shown in Figs. 20a and 20b. These are two orders of magnitudes smaller than the transmission coefficients and would hardly be visible plotting all coefficients simultaneously.

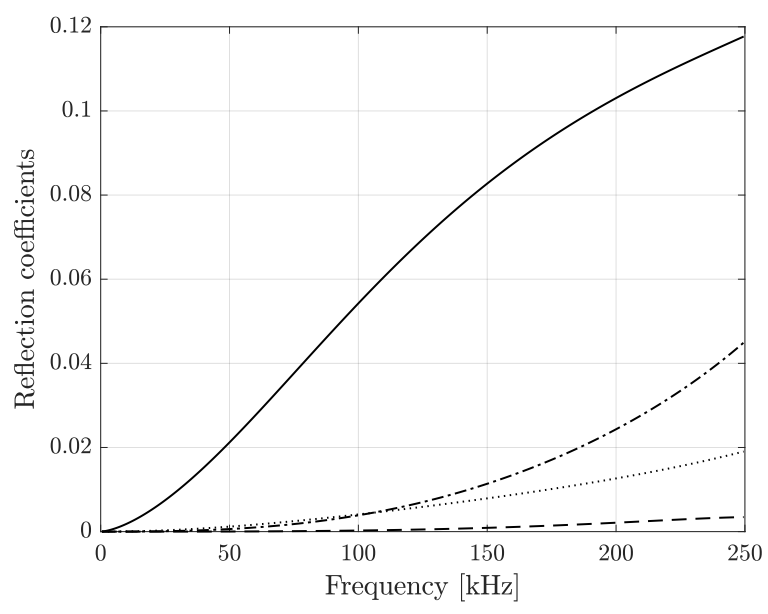

(a) Reflection coefficient

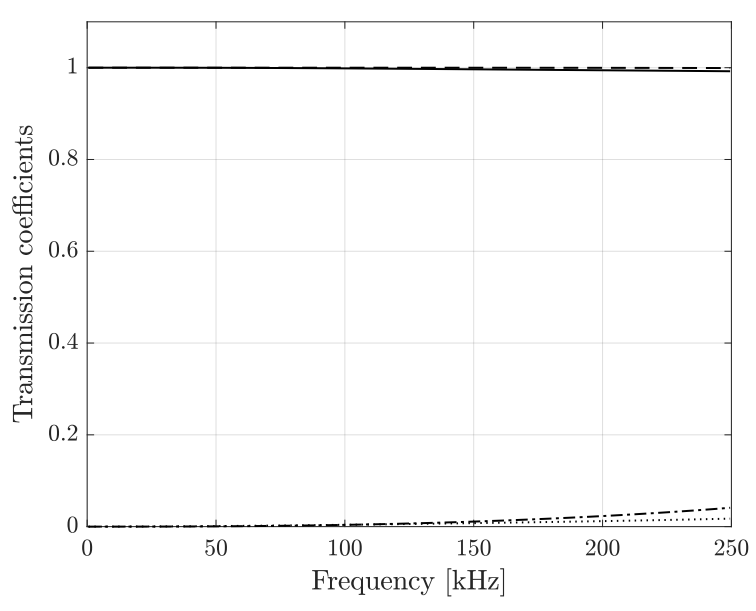

(b) Transmission coefficient

Figure 19: Scattering coefficients for a delamination in a composite beam. $\mathrm{S}_{0}$ to $\mathrm{S}_{0}(--), \mathrm{S}_{0}$ to $\mathrm{A}_{0}(\cdots), \mathrm{A}_{0}$ to $\mathrm{A}_{0}(-)$ and $\mathrm{A}_{0}$ to $S_{0}(-\cdot-)$.

The mesh for the FE simulation contains 72000 C3D8R elements. The simulated time window is $300 \mu \mathrm{s}$ because of the lower group velocity of $\mathrm{A}_{0}$ mode. The time taken for the simulation is 2465s. After loading necessary data into the memory, the reconstruction takes $0.2192 \mathrm{~s}$. The results from FE and WFE approach are shown in Figs. 21 and 22 for the in-plane and out-of-plane displacements respectively. Fig. 21a shows three different reflections from damage. The mode converted $\mathrm{S}_{0}$ waves are faster, so they reach the observation point 1 first. Their amplitude is much smaller compared to the reflected $\mathrm{A}_{0}$ wave which arrives later. The amplitude of the reflected $\mathrm{S}_{0}$ wave is so small that it results in negligible out-of-plane displacements in Fig. 21b. The same holds for observation point 2 where only two waves are observed in the simulated 


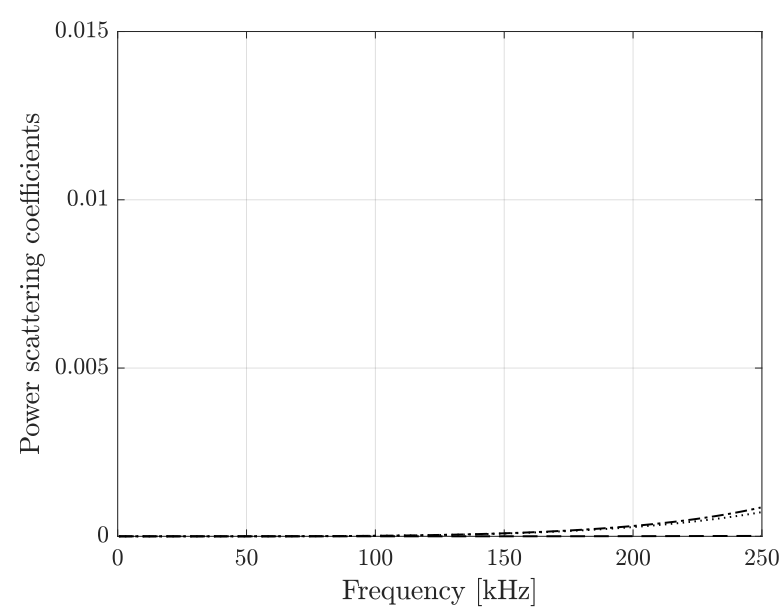

(a) Incident $\mathrm{S}_{0}$ wave

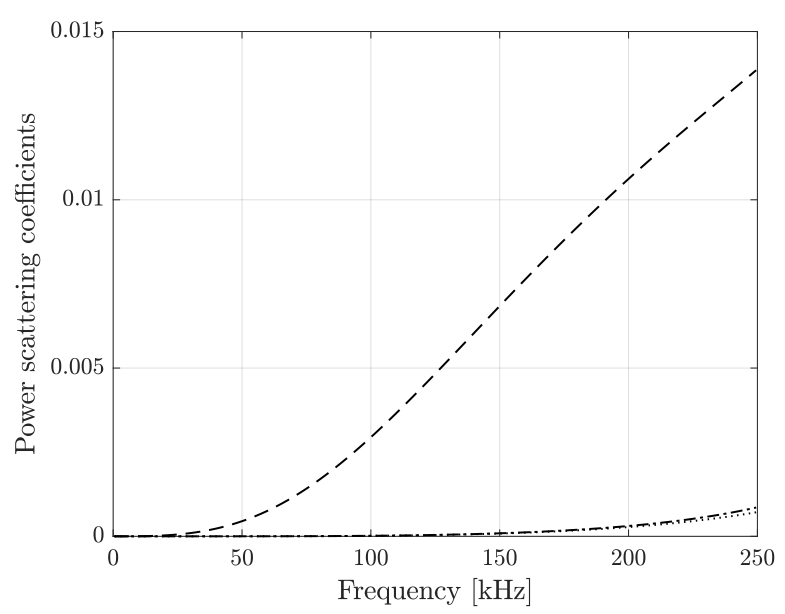

(b) Incident $A_{0}$ wave

Figure 20: Energy carrying modes for a beam with a delamination with $S_{0}$ to $S_{0}$ and $A_{0}$ to $A_{0}$ reflection coefficients (- -), $S_{0}$ to $A_{0}$ and $A_{0}$ to $S_{0}$ reflection (---) and transmission coefficients $(\cdots) . S_{0}$ to $S_{0}$ and $A_{0}$ to $A_{0}$ transmission coefficients are not plotted as they are an order of magnitude larger.

time window, a mode converted $S_{0}$ wave and a $A_{0}$ wave. The results are once again found to be in good agreement with each other.

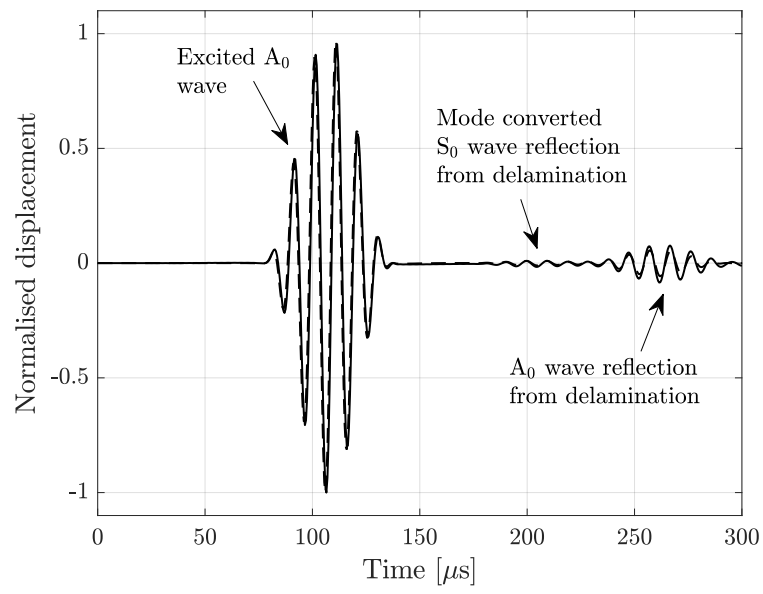

(a) In-plane

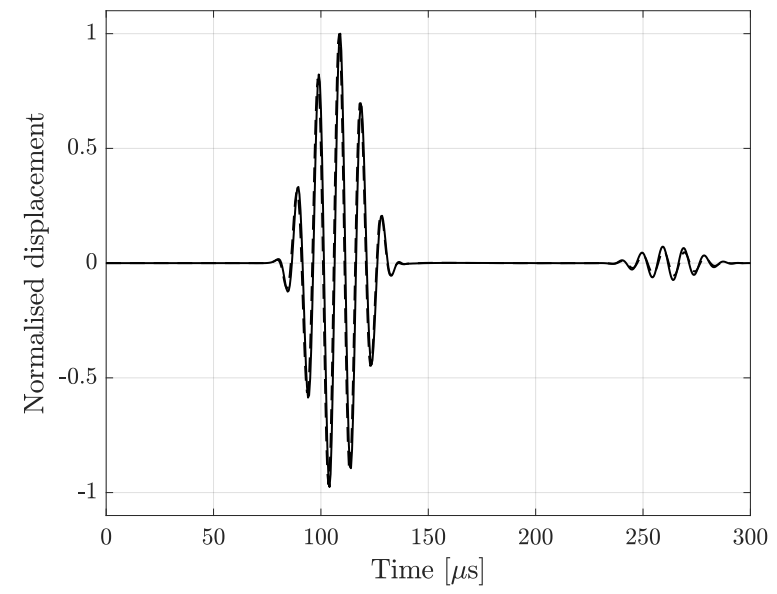

(b) Out-of-plane

Figure 21: Time domain comparison between FE (-) and WFE (- -) at a node located at observation point 1 for a composite beam with a delamination damage. 


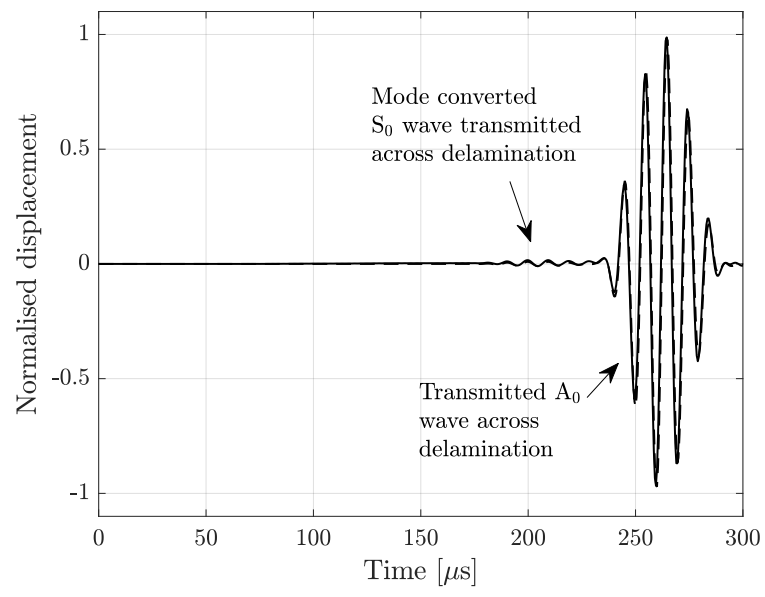

(a) In-plane

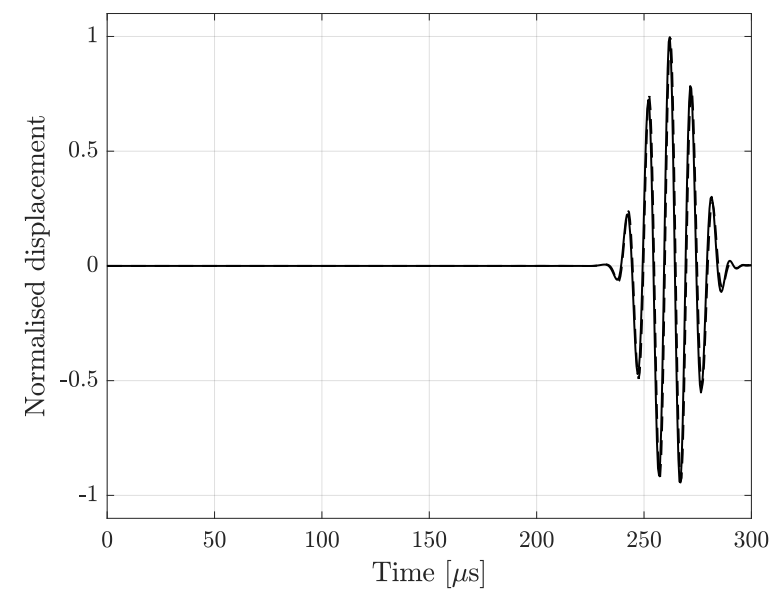

(b) Out-of-plane

Figure 22: Time domain comparison between FE (-) and WFE (- -) at a node located at observation point 2 for a composite beam with a delamination damage.

\section{Conclusions}

An approach for transient simulations of ultrasonic guided waves in layered composite structures is presented. A number of different scenarios of wave propagation and damage types are modelled and validated to support the claims made. The main conclusions of the work are as follows:

- An approach for applying complex displacement fields which are representative of physical excitation from piezoelectric transducers is developed in the scope of guided wave excitation in structures.

- An approach for modelling any shape or type of damage is integrated in the methodology to model actual physical damage scenarios.

- A robust model order reduction strategy has been developed to select only those modes which contribute towards the final transient response in the structure.

- An excellent agreement is observed between the WFE approach and full FE simulations.

- Orders of magnitude reduction in computational time and memory consumption is observed comparing WFE to FE simulations. It should be noted that this reduction will be even more pronounced when the approach is programmed in an optimal solver.

Considering the strengths of this approach it can be used to train surrogate models for generating a comprehensive library of results for multiple damage scenarios. This may become part of a probabilistic SHM framework for damage detection and identification. A natural next step is to extend this work to 
two dimensional composite structures where performing full FE simulations for multiple scenarios no longer remains practical due to large computational times.

\section{Acknowledgements}

This work has been done as part of the SAFE-FLY project funded by the European Union Horizon 2020 Research and Innovation Programme under the Marie Sklodowska-Curie grant agreement No 721455.

\section{Data availability}

The raw/processed data required to reproduce these findings cannot be shared at this time as the data also forms part of an ongoing study. They will be made available upon request.

\section{References}

[1] G. Hellard, Composites in Airbus: A long story of innovations and experiences, in: EADS Global Investor Forum, Sevilla, 2008.

[2] J. Hale, Boeing 787 from the ground up, Aero 4 (24) (2006) 7.

[3] R. Talreja, C. V. Singh, Damage and failure of composite materials, Cambridge University Press, 2012.

[4] O. Büyüköztürk, Imaging of concrete structures, NDT \& E International 31 (4) (1998) 233-243.

[5] V. Giurgiutiu, Structural health monitoring of aerospace composites, Academic Press, Oxford, 2015.

[6] J. Krautkramer, H. Krautkramer, Ultrasonic testing of materials, the 4th edition, Springer Verlag, Berlin, 1990.

[7] B. Helifa, A. Oulhadj, A. Benbelghit, I. Lefkaier, F. Boubenider, D. Boutassouna, Detection and measurement of surface cracks in ferromagnetic materials using eddy current testing, NDT \& E International 39 (5) (2006) 384-390.

[8] S. Banerjee, F. Ricci, E. Monaco, A. Mal, A wave propagation and vibration-based approach for damage identification in structural components, Journal of Sound and Vibration 322 (1-2) (2009) 167-183.

[9] R. Gangadharan, D. R. Mahapatra, S. Gopalakrishnan, C. Murthy, M. Bhat, On the sensitivity of elastic waves due to structural damages: Time-frequency based indexing method, Journal of Sound and Vibration 320 (4-5) (2009) 915-941.

[10] D. Aggelis, T. Matikas, Effect of plate wave dispersion on the acoustic emission parameters in metals, Computers \& Structures 98 (2012) 17-22.

[11] J. L. Rose, A vision of ultrasonic guided wave inspection potential, in: Proceedings of the 7th ASME NDE Tropical Conference, 2001, pp. 1-22.

[12] G. Aranguren, P. Monje, V. Cokonaj, E. Barrera, M. Ruiz, Ultrasonic wave-based structural health monitoring embedded instrument, Review of Scientific Instruments 84 (12) (2013) 125106.

[13] M. Ruiz González, P. M. Monje, L. Casado, G. Aranguren, V. Cokonaj, E. Barrera Lopez de Turiso, Integrated electronic system for ultrasonic structural health monitoring, in: European workshop on structural health monitoring, 2012, pp. 1-8.

[14] A. Alcaide, E. Barrera, M. Ruiz, G. Aranguren, Damage detection on aerospace structures using pamela shm system, in: 6th International Symposium on NDT in Aerospace, Madrid, 2014.

[15] C. Farrar, K. Worden, Structural Health Monitoring: A Machine Learning Perspective, John Wiley and Sons, 2012.

[16] S. Cantero-Chinchilla, J. Chiachío, M. Chiachío, D. Chronopoulos, A. Jones, A robust bayesian methodology for damage localization in plate-like structures using ultrasonic guided-waves, Mechanical Systems and Signal Processing 122 (2019) $192-205$. 
[17] H. Fukunaga, N. Hu, F.-K. Chang, Structural damage identification using piezoelectric sensors, International Journal of Solids and Structures 39 (2) (2002) 393-418.

[18] Y.-D. Xu, S.-f. Yuan, G. Peng, Study on two-dimensional damage location in structure based on active lamb wave detection technique, Journal of Vibration Engineering 25 (5) (2004) 476-479.

[19] J. E. Michaels, T. E. Michaels, Guided wave signal processing and image fusion for in situ damage localization in plates, Wave Motion 44 (6) (2007) 482-492.

[20] R. Monkhouse, P. Wilcox, M. Lowe, R. Dalton, P. Cawley, The rapid monitoring of structures using interdigital lamb wave transducers, Smart Materials and Structures 9 (3) (2000) 304-309.

[21] K. F. Graff, Wave motion in elastic solids, Dover Publications, New York, 2012.

[22] T. Hayashi, K. Kawashima, J. L. Rose, Calculation for guided waves in pipes and rails, in: Key Engineering Materials, Vol. 270, Trans Tech Publ, 2004, pp. 410-415.

[23] I. Bartoli, A. Marzani, F. L. di Scalea, E. Viola, Modeling wave propagation in damped waveguides of arbitrary crosssection, Journal of Sound and Vibration 295 (3-5) (2006) 685-707.

[24] Z. Ahmad, J. Vivar-Perez, U. Gabbert, Semi-analytical finite element method for modeling of lamb wave propagation, CEAS Aeronautical Journal 4 (1) (2013) 21-33.

[25] Y. Shen, Structural health monitoring using linear and nonlinear ultrasonic guided waves, Ph.D. thesis, University of South Carolina (2014).

[26] V. Giurgiutiu, M. Gresil, B. Lin, A. Cuc, Y. Shen, C. Roman, Predictive modeling of piezoelectric wafer active sensors interaction with high-frequency structural waves and vibration, Acta Mechanica 223 (8) (2012) 1681-1691.

[27] V. Thierry, L. Brown, D. Chronopoulos, Multi-scale wave propagation modelling for two-dimensional periodic textile composites, Composites Part B: Engineering 150 (2018) 144-156.

[28] H. Gravenkamp, C. Birk, C. Song, Simulation of elastic guided waves interacting with defects in arbitrarily long structures using the scaled boundary finite element method, Journal of Computational Physics 295 (2015) 438-455.

[29] B. R. Mace, E. Manconi, Modelling wave propagation in two-dimensional structures using finite element analysis, Journal of Sound and Vibration 318 (4-5) (2008) 884-902.

[30] D. Chronopoulos, C. Droz, R. Apalowo, M. Ichchou, W. Yan, Accurate structural identification for layered composite structures, through a wave and finite element scheme, Composite Structures 182 (2017) 566-578.

[31] R. Apalowo, D. Chronopoulos, G. Tanner, Wave interaction with defects in pressurised composite structures, Journal of Nondestructive Evaluation 37 (3) (2018) 48.

[32] D. Chronopoulos, Wave steering effects in anisotropic composite structures: Direct calculation of the energy skew angle through a finite element scheme, Ultrasonics 73 (2017) 43-48.

[33] M. Mallouli, M. B. Souf, O. Bareille, M. Ichchou, T. Fakhfakh, M. Haddar, Transient wave scattering and forced response analysis of damaged composite beams through a hybrid finite element-wave based method, Finite Elements in Analysis and Design 147 (2018) 1-9.

[34] M. Mallouli, M. B. Souf, O. Bareille, M. Ichchou, T. Fakhfakh, M. Haddar, Damage detection on composite beam under transverse impact using the wave finite element method, Applied Acoustics 147 (2019) 23-31.

[35] Y. Shen, V. Giurgiutiu, Effective non-reflective boundary for lamb waves: Theory, finite element implementation, and applications, Wave Motion 58 (2015) 22-41.

[36] J. M. Renno, B. R. Mace, Calculation of reflection and transmission coefficients of joints using a hybrid finite element/wave and finite element approach, Journal of Sound and Vibration 332 (9) (2013) 2149-2164.

[37] Y. Waki, B. Mace, M. Brennan, Numerical issues concerning the wave and finite element method for free and forced vibrations of waveguides, Journal of Sound and Vibration 327 (1-2) (2009) 92-108.

[38] J. F. Doyle, Wave propagation in structure: spectral analysis using fast discrete Fourier transforms, Springer Verlag, 
NewYork, 1997.

[39] Y. Waki, On the application of finite element analysis to wave motion in one-dimensional waveguides, Ph.D. thesis, University of Southampton (2007).

[40] W. Zhong, F. Williams, On the direct solution of wave propagation for repetitive structures, Journal of Sound and Vibration 181 (3) (1995) 485-501.

[41] D. Chronopoulos, Calculation of guided wave interaction with nonlinearities and generation of harmonics in composite structures through a wave finite element method, Composite Structures 186 (2018) 375-384.

[42] J. L. Rose, Ultrasonic guided waves in solid media, Cambridge University Press, 2014.

[43] B. R. Mace, D. Duhamel, M. J. Brennan, L. Hinke, Finite element prediction of wave motion in structural waveguides, The Journal of the Acoustical Society of America 117 (5) (2005) 2835-2843.

[44] G. Mitrou, N. Ferguson, J. Renno, Wave transmission through two-dimensional structures by the hybrid FE/WFE approach, Journal of Sound and Vibration 389 (2017) 484-501.

[45] ABAQUS, Abaqus Documentation, Dassault Systèmes, Providence, RI, USA (2016).

[46] Z. Su, L. Ye, Y. Lu, Guided lamb waves for identification of damage in composite structures: A review, Journal of Sound and Vibration 295 (3-5) (2006) 753-780. 